ARTICLE

https://doi.org/10.1038/s41467-019-14076-3

\title{
Endothelial ZEB1 promotes angiogenesis- dependent bone formation and reverses osteoporosis
}

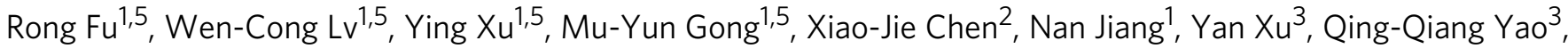 \\ Lei $\mathrm{Di}^{1}$, Tao $\mathrm{Lu}^{4}$, Li-Ming Wang ${ }^{3}$, Ran $\mathrm{Mo}^{2} \&$ Zhao-Qiu Wu ${ }^{1 *}$
}

Recent interest in the control of bone metabolism has focused on a specialized subset of CD31 hi endomucin ${ }^{\text {hi }}$ vessels, which are reported to couple angiogenesis with osteogenesis. However, the underlying mechanisms that link these processes together remain largely undefined. Here we show that the zinc-finger transcription factor ZEB1 is predominantly expressed in $\mathrm{CD} 31^{\text {hi }}$ endomucin ${ }^{\text {hi }}$ endothelium in human and mouse bone. Endothelial cellspecific deletion of ZEB1 in mice impairs CD31 hiendomucin ${ }^{\text {hi }}$ vessel formation in the bone, resulting in reduced osteogenesis. Mechanistically, ZEB1 deletion reduces histone acetylation on DII4 and Notch1 promoters, thereby epigenetically suppressing Notch signaling, a critical pathway that controls bone angiogenesis and osteogenesis. ZEB1 expression in skeletal endothelium declines in osteoporotic mice and humans. Administration of Zeb1-packaged liposomes in osteoporotic mice restores impaired Notch activity in skeletal endothelium, thereby promoting angiogenesis-dependent osteogenesis and ameliorating bone loss. Pharmacological reversal of the low ZEB1/Notch signaling may exert therapeutic benefit in osteoporotic patients by promoting angiogenesis-dependent bone formation.

\footnotetext{
${ }^{1}$ State Key Laboratory of Natural Medicines, School of Basic Medicine and Clinical Pharmacy, China Pharmaceutical University, Nanjing 211198, China. ${ }^{2}$ State Key Laboratory of Natural Medicines, Jiangsu Key Laboratory of Drug Discovery for Metabolic Diseases, Center of Advanced Pharmaceuticals and Biomaterials, China Pharmaceutical University, Nanjing 210009, China. ${ }^{3}$ Department of Orthopedic Surgery, Digital Medicine Institute, The Affiliated Nanjing Hospital of Nanjing Medical University, Nanjing 210006, China. ${ }^{4}$ State Key Laboratory of Natural Medicines, Laboratory of Molecular Design and Drug Discovery, School of Science, China Pharmaceutical University, Nanjing 211198, China. ${ }^{5}$ These authors contributed equally: Rong Fu, Wen-Cong Lv, Ying Xu, Mu-Yun Gong. *email: zqwu@cpu.edu.cn
} 
A specific vessel subtype, strongly positive for CD31 and endomucin (CD31 ${ }^{\text {hi }}$ EMCN $\left.{ }^{\text {hi }}\right)$, has been recently reported to couple angiogenesis with osteogenesis in the bone ${ }^{1-4}$. CD31 ${ }^{\text {hiEMCN }}{ }^{\text {hi }}$ vessels, residing in the bone marrow near the growth plate, represent the key component of a metabolically specialized bone microenvironment with privileged access to nutrients and oxygen, thereby promoting the growth potential of surrounding perivascular cells, including pre-osteoprogenitors, osteoprogenitors, mature osteoblasts, mature and hypertrophic chondrocytes, and hematopoietic stem cells ${ }^{1-5}$. It is increasingly appreciated that skeletal $\mathrm{CD} 31^{\text {hi }} \mathrm{EMCN}{ }^{\text {hi }}$ vessel numbers decline in ageing and ovariectomy (OVX)-induced osteoporotic mice and in osteoporotic patients ${ }^{1,4,6-9}$. Osteoporosis is a systemic bone disease characterized by abnormal bone metabolism, low bone mass and density, impaired bone microstructure, and increased bone fragility and fracture prone ${ }^{6-9}$. Reduced $\mathrm{CD} 31^{\text {hi }} \mathrm{EMCN} \mathrm{N}^{\mathrm{hi}}$ endothelium levels in the bone are strongly associated with osteoporosis progression. Thus, pharmacological restorage of $\mathrm{CD} 31^{\text {hiEMCN }}{ }^{\text {hi }}$ endothelium levels in the bone may exert therapeutic benefit in osteoporotic patients by promoting angiogenesis-dependent osteogenesis. The evolutionarily conserved Dll4/Notch signaling in the bone endothelium has been reported to increase $\mathrm{CD} 31^{\text {hi }} \mathrm{EMCN}^{\mathrm{hi}}$ endothelium levels and thus promote bone formation by upregulating secretion levels of Noggin, a Notch-controlled angiocrine factor ${ }^{2}$. However, it is currently unclear how Notch activity is tightly controlled in the bone endothelium and whether declined endothelial Notch signaling is linked to osteoporosis.

Zinc-finger E-box-binding homeobox 1 (ZEB1) is most frequently characterized as a zinc-finger transcription factor that triggers the epithelial-mesenchymal transition (EMT) programs critical to development as well as a range of pathologic states, including cancer ${ }^{10-13}$. Previous studies have demonstrated that global deletion of ZEB1 in mice elicits perinatal lethality due to severely skeletal defects ${ }^{14}$. However, the primary cell lineage(s) affected in these mice and the underlying molecular mechanisms remain undefined. In addition, it remains unclear whether ZEB1 is required for postnatal bone formation or whether ZEB1 loss in certain cell lineage(s) is linked to osteoporotic disease in human and mice. Here we show that ZEB1 is predominantly expressed in CD31 hiEMCN ${ }^{\text {hi }}$ endothelium in human and mouse bone, and ZEB1 levels decline in ageing and OVX-induced osteoporotic mice, as well as in osteoporotic patients. Endothelial cell (EC)specific deletion of ZEB1 in mice reduces CD31 hiEMCN ${ }^{\text {hi }}$ vascularization and osteogenesis by epigenetically repressing Dll4/ Notch signaling. Administration of recombinant Dll4 protein efficiently restores CD31 hiEMCN ${ }^{\text {hi }}$ vessel growth and bone formation in ZEB1-deleted mice. Importantly, we observe low ZEB1Dll4/Notch signaling in OVX-induced osteoporotic mice and in vivo $Z E B 1$ gene delivery restores impaired Notch signaling, thereby boosting CD31 hiEMCN ${ }^{\text {hi }}$ vessel formation, promoting osteogenesis, and ameliorating bone loss in OVX-induced osteoporotic mice. In summary, our results lay the foundation for new therapeutic strategies in osteoporosis treatment by promoting angiogenesis-dependent bone formation.

\section{Results}

ZEB1 is predominantly expressed in CD31 ${ }^{\text {hi}} \mathrm{EMCN}^{\text {hi }}$ bone ECs. We scanned tissues that were collected from juvenile 3-week-old mice for ZEB1 protein expression. Interestingly, we found that ZEB1, as detected by immunofluorescence, was expressed in the endothelium of skeletal elements such as the tibia, sternum, and vertebra at significantly higher positivity and expression levels than in the endothelium of non-skeletal organs such as the spleen, lung, kidney, liver, and heart (Fig. 1a, b). Further, we observed that ZEB1 protein was predominantly expressed in metaphyseal CD31 hiEMCNhi (termed as type $\mathrm{H}$ ) endothelium of tibia, while it was essentially undetectable in the CD $31^{\text {low }}{ }^{2} M C N^{\text {low }}$ (termed as type L) endothelium found within the bone marrow (Fig. 1c, d). These observations suggest a markedly distinct ZEB1 expression pattern between type $\mathrm{H}$ and $\mathrm{L}$ vessels in mouse long bone (e.g., tibia), a finding that could be extended to other skeletal elements such as the sternum, calvarium, and vertebra (Fig. 1c, d). Importantly, the distinct ZEB1 expression pattern observed in mouse bone was also presented in human tibia (Fig. 1c, d). Furthermore, we performed a quantitative reverse transcription PCR (RT-qPCR) assay on fluorescence-activated cell (FACS)sorted type H vs. type L tibial ECs of 3-week-old mice. The results demonstrated that Zeb1 transcript levels in type $\mathrm{H}$ tibial ECs were also significantly higher than in type L ECs (Fig. 1e). Intriguingly, transcript levels of Zeb2, another member of ZEB family, in type $\mathrm{H}$ bone ECs were remarkably lower than in type L ECs, an expression pattern that is highly distinct from ZEB1 in bone ECs (Fig. 1e). It has been previously reported that type $\mathrm{H}$ vessel numbers and bone mass decline in tandem with ageing in human and murine skeletal system ${ }^{1,6}$ (Fig. 1f-h). Notably, ZEB1 protein in type $\mathrm{H}$ tibial ECs was remarkably more abundant in juvenile (3-week-old) mice relative to (10-week-old) adults and was largely absent in aged (50-week-old) animals (Fig. 1h, i), suggesting that ZEB1 protein is dynamically controlled during ageing. On the basis of the specific expression pattern of ZEB1 in the skeletal system, we considered the possibility that ZEB1 expressed by type $\mathrm{H}$ bone ECs functions as a key signaling regulator of angiogenesis and bone formation.

Endothelial ZEB1 deletion reduces type $\mathrm{H}$ vessel formation. To investigate ZEB1 function in the bone endothelium, conditional Zeb1 floxed/floxed $\left(Z e b 1^{f l f l}\right)$ mice were generated in our laboratory (Supplementary Fig. 1a; ref. ${ }^{15}$ ) and crossed with Tie2-Cre ${ }^{16,17}$ transgenic line to generate Tie2-Cre $e^{-} ; \mathrm{Zeb} 1^{f l f l}$ control and Tie2-Cre ${ }^{+} ; Z e b 1^{f l f l}$ EC-specific ZEB1 knockout mice (designated $Z e b 1^{W T}$ and $Z e b 1^{\Delta E C}$ mice, respectively). In addition, Zeb1 $1^{f l / f l}$ mice were also mated with $C d h 5(P A C)-C r e E R T 2^{18}$ transgenic line and the resulting $C d h 5(P A C)-C r e E R T 2^{-} ; Z e b 1^{f l / f l}$ and $C d h 5(P A C)-$ CreERT2 ${ }^{+} ; Z e b 1^{\text {flf } f l}$ mice were treated with tamoxifen to generate control and EC-specific ZEB1 knockout mice (designated Zeb1 ${ }^{W T}$ and Zeb1 ${ }^{i \Delta E C}$ mice, respectively). RT-qPCR analysis of FACSsorted tibial ECs revealed significantly reduced Zeb1 transcript levels in 3-week-old $Z e b 1^{\triangle E C}$ compared with control littermates (Supplementary Fig. 1b, c). Also, Zeb1 transcript levels were remarkably decreased in tibial ECs of 3-week-old Zebi $1^{i \Delta E C}$ mice that were intraperitoneally (i.p.) injected with $0.1 \mathrm{mg}$ tamoxifen every day at postnatal day 8 (P8) for 7 consecutive days, as compared with Zeb1 ${ }^{W T}$ control mice receiving identical tamoxifen treatment (Supplementary Fig. 1c). Furthermore, immunofluorescence analysis of tibial sections revealed that ZEB1 protein was efficiently depleted in type $\mathrm{H}$ bone ECs but not perivascular cells of 3-week-old Zeb1 $1^{\triangle E C}$ mice (Supplementary Fig. 1d, e) and Zeb1 ${ }^{i \triangle E C}$ mice (Supplementary Fig. 1f, g). Constitutive and tamoxifen inducible inactivation of endothelial ZEB1 in 3-weekold mice markedly decreased the density of type $\mathrm{H}$ vessels, but not type L vessels, in skeletal elements such as tibia, sternum, vertebra, and calvarium, as assessed by immunofluorescence (Fig. 2a-c and Supplementary Fig. 2a, b) and FACS (Fig. 2d, e) analyses. Similarly, 10-week-old adult Zeb1 $1^{\triangle E C}$ mice and Zeb1 ${ }^{i \Delta E C}$ mice that were i.p. injected with $1.0 \mathrm{mg}$ tamoxifen every other day at 7 weeks of ages for 2 consecutive weeks both exhibited substantially reduced type $\mathrm{H}$ but not type L vessel density compared with their corresponding littermate controls (Supplementary Fig. 2c-f). By contrast, Zeb1 ${ }^{\triangle E C}$ mice exhibited comparable $\mathrm{CD} 31^{+}$vessel densities in non-skeletal tissues such as the heart, 
a

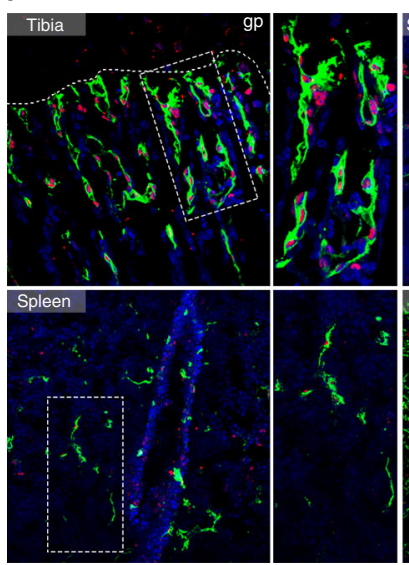

C
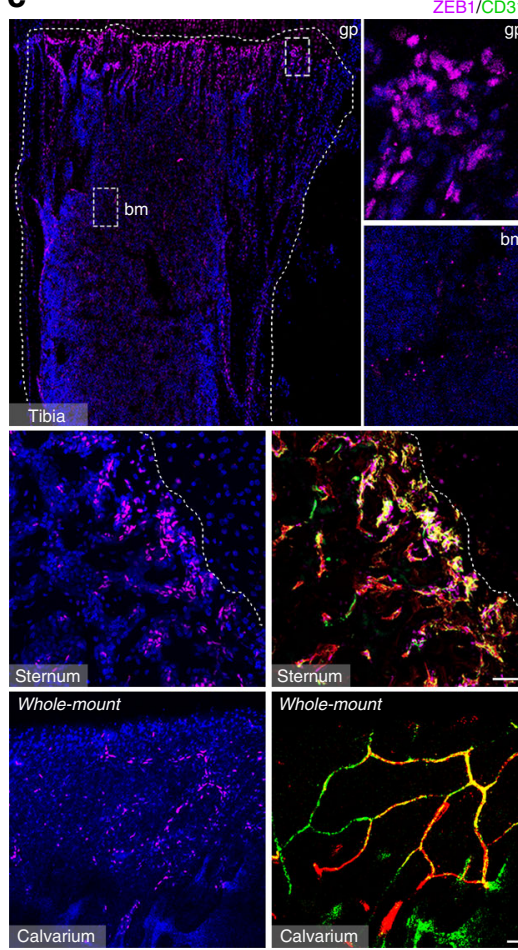

g
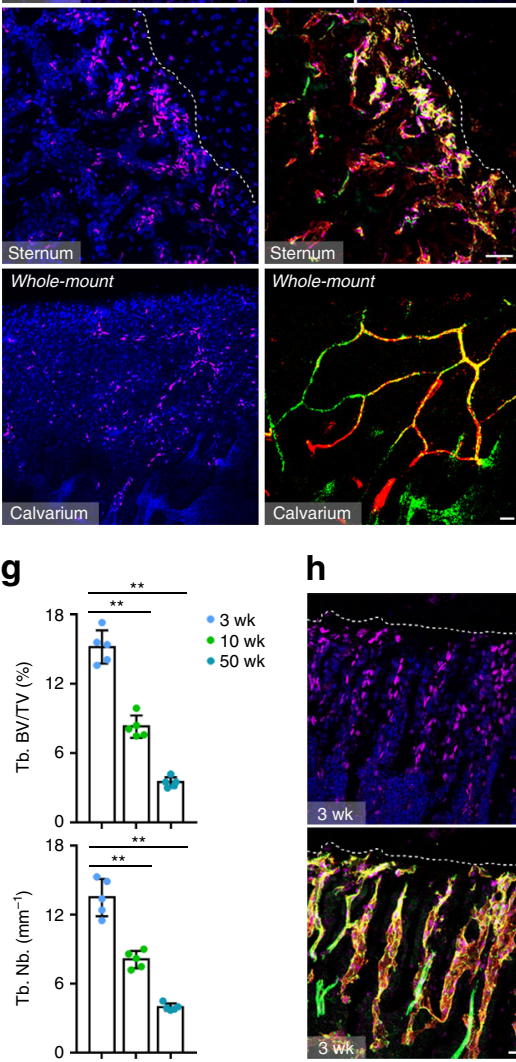

h
ZEB1/CD31/DAP

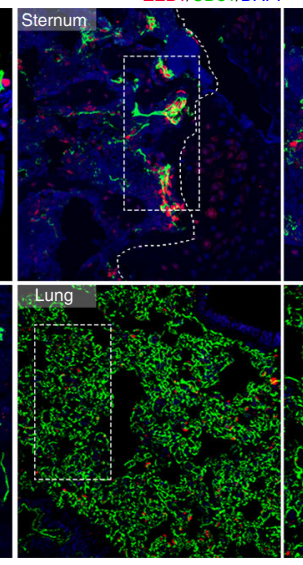

ZEB1/CD31/EMCN/DAPI
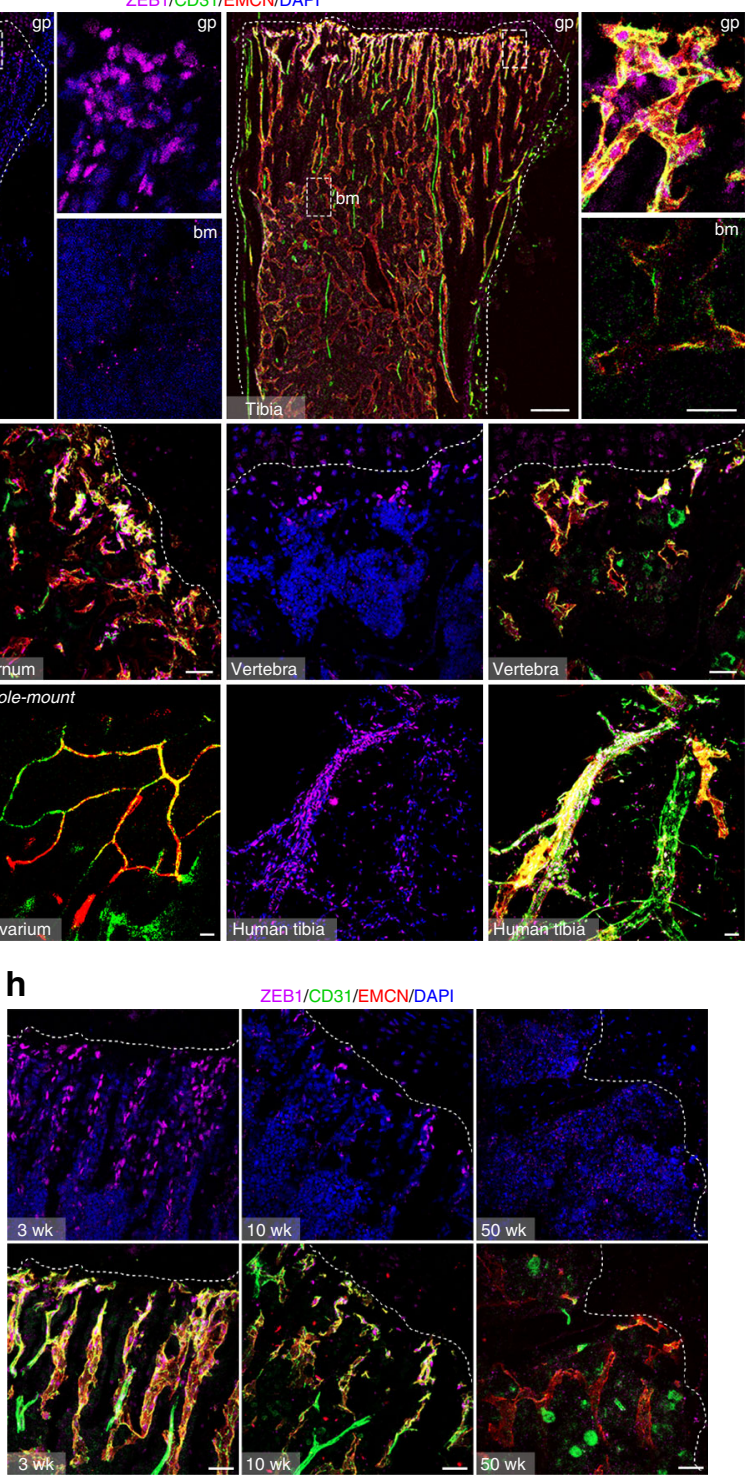

b

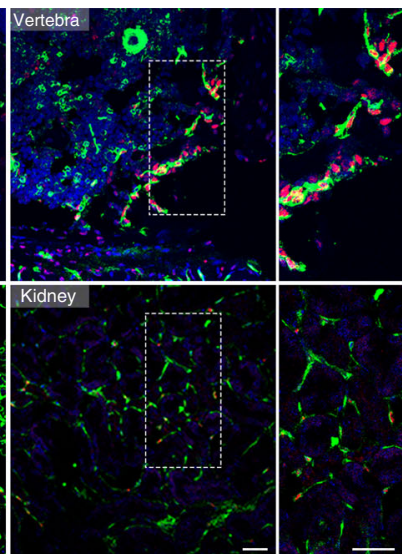

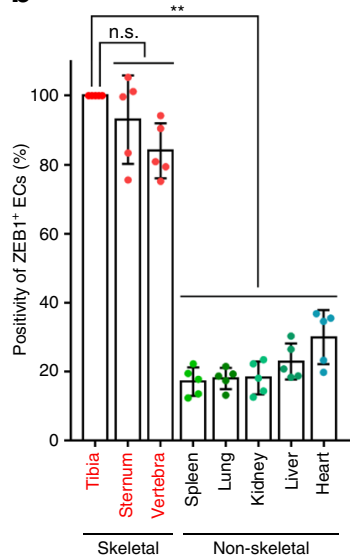

d

d Type H - Type L

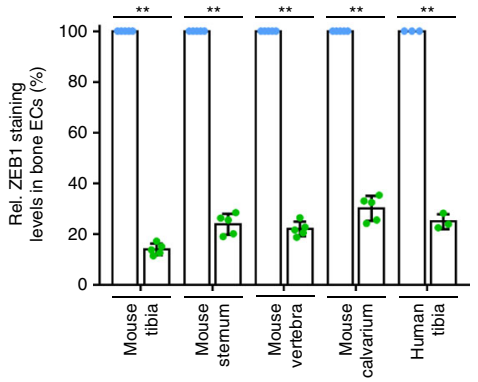

e

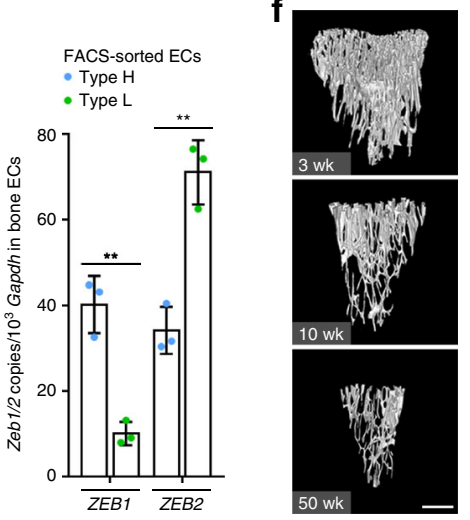

i

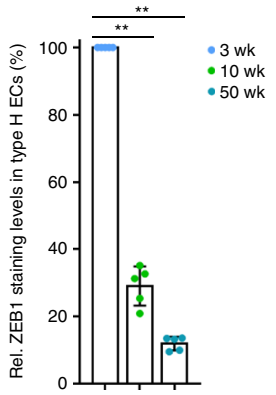

lung, liver, kidney, and spleen relative to littermate controls (Supplementary Fig. 2g, h). Vascular endothelial growth factor A (VEGFA), a potent proangiogenic growth factor secreted by endothelial column/arches, perivascular cells, and mature/ hypertrophic chondrocytes ${ }^{19}$, was robustly decreased in the tibia of 3-week-old Zeb1 ${ }^{\triangle E C}$ mice, as assessed by immunofluorescence analysis (Fig. 2f, g). RT-qPCR analysis of FACS-sorted tibial ECs also revealed a strong reduction in Vegfa transcript levels in 3week-old Zeb1 $1^{\triangle E C}$ mice compared with littermate controls (Fig. 2h). These findings are consistent with previous reports demonstrating that ZEB1 is positively associated with VEGFA expression in cancer cells ${ }^{20}$ and cancer-associated fibroblasts ${ }^{15}$. 
Fig. 1 ZEB1 is primarily expressed in type $\mathbf{H}$ vessels of human and murine bone. a Representative confocal images showing ZEB1 expression in CD31+ ECs of the tibia, sternum, vertebra, spleen, lung, and kidney dissected from juvenile 3-week-old mice $(n=5)$. Nuclei, 4',6-diamidino-2-phenylindole (DAPI). Magnified areas of dashed boxed sections are shown in right panels. gp growth plate. Scale bar, $30 \mu \mathrm{m}$. b Quantification of positivity of ZEB1+ ECs as shown in a ( $n=5$ independent experiments). c Comparisons of ZEB1 staining levels in CD31hiEMCN hi (type H) vs. CD31 ${ }^{\text {loEMCN }}{ }^{\text {lo }}$ (type L) ECs of 3-weekold tibia, sternum, vertebra, and calvarium $(n=5)$, and human tibia $(n=3)$. Magnified areas of dashed boxed sections in top row are shown in right panels. bm bone marrow, EMCN endomucin. Scale bar: $30 \mu \mathrm{m}, 200 \mu \mathrm{m}$ (top row, lower magnification images). d Quantification of ZEB1 staining levels in two types of bone ECs as shown in c ( $n=5$ or 3 independent experiments). e RT-qPCR analysis of Zeb1 and Zeb2 transcripts in FACS-sorted type H and type L bone ECs of 3-week-old mice ( $n=3$ independent experiments). f Representative micro-CT images of trabecular bone of 3-, 10-, and 50-week-old tibia ( $n=5$ ). Scale bar, $0.2 \mathrm{~mm}$. $\mathbf{g}$ Quantification of trabecular bone fraction (Tb. BV/TV; trabecular bone, bone volume/tissue volume) and trabecular number (Tb. Nb.) in tibia as shown in $\mathbf{f}(n=5$ independent experiments). $\mathbf{h}$ Representative confocal images of ZEB1, CD31, and EMCN immunostained sections of 3-, 10- or 50 -week-old tibia ( $n=5$, each). Scale bar, $30 \mu \mathrm{m}$. i Quantification of ZEB1 staining levels in tibial type H ECs as shown in $\mathbf{h}$ ( $n=5$ independent experiments). All data are represented as mean \pm SD. ${ }^{\star \star} P<0.01$; NS not significant. Differences are tested using one-way ANOVA with Tukey's post hoc test $(\mathbf{b}, \mathbf{g}, \mathbf{i})$ and unpaired two-tailed Student's t-test $(\mathbf{d}, \mathbf{e})$. The source data are provided as a Source Data file.

Microphil-perfused angiography demonstrated a markedly reduced vessel number and volume in the metaphysis of 3-weekold Zeb1 ${ }^{\triangle E C}$ tibia (Fig. 2i) where disrupted column/arch patterning and impaired filopodia extension were observed (Fig. 2j, k).

Endothelial ZEB1 deletion impairs bone formation. Severely defective angiogenesis in the skeletal organs of $Z e b 1^{\Delta E C}$ mice at 3, 10 , and 50 weeks of ages was accompanied by significantly reduced body weight and shortened femoral length (Fig. 3a and Supplementary Fig. 3a). Micro-computed tomography (microCT) and histo-morphometric analyses revealed significant loss of trabecular bone density (trabecular bone volume per tissue volume (Tb. BV/TV)), trabecular number (Tb. Nb.), trabecular thickness (Tb. Th.), and cortical thickness (Ct. Th.) in the tibia of 3-, 10-, and 50-week-old Zeb1 ${ }^{\triangle E C}$ mice (Fig. 3b, c and Supplementary Fig. 3b-d). By contrast, no structural alterations were detected in 3-week-old $Z e b 1^{\triangle E C}$ non-skeletal organs such as the heart, liver, spleen, lung, and kidney (Supplementary Fig. 3e). Notably, 3-week-old Zeb1 $1^{i \triangle E C}$ mice also exhibited markedly reduced body weight (Supplementary Fig. 3f, left two columns) in tandem with severely impaired bone formation (Supplementary Fig. $3 g, h)$. As body weight is believed to have a profound impact on bone mass, we further assessed whether impaired bone formation observed in endothelial ZEB1-deleted mice was due to reduced body weight of the mice. To this end, we induced endothelial ZEB1 deletion in mice at 7 weeks of ages, a stage that skeletal growth has been completed. As shown, body weight of 10 -week-old adult $Z e b 1^{W T}$ and $Z e b 1^{i \Delta E C}$ mice was largely comparable (Supplementary Fig. 3f, right two columns), whereas bone mass of Zeb1 $1^{i \triangle E C}$ mice was significantly lower than $Z e b 1^{W T}$ mice, as assessed by micro-CT analysis (Supplementary Fig. 3i, j). Thus, these findings demonstrate that endothelial ZEB1 deletion at young or adult ages both elicits impairment of bone formation independently of body weight reduction in the mice. Calcein double labeling further confirmed strongly reduced bone formation rates in the tibia of 3 -week-old $Z e b 1^{\triangle E C}$ mice (Fig. $2 \mathrm{~d}$, e). Importantly, 3-week-old Zeb1 ${ }^{\triangle E C}$ and $Z e b 1^{i \Delta E C}$ mice showed significantly reduced numbers of Runx $2^{+}$pre-osteoprogenitors ${ }^{21}$, Osterix $^{+}$osteoprogenitors $^{22}$, as well as $\mathrm{ALP}^{+}$mature osteoblasts $^{23}$ in tandem with impaired mineralized bone mass (Alizarin staining), but without appreciable changes in the TRAP ${ }^{+}$ preosteoclasts numbers ${ }^{24}$ (Fig. $3 \mathrm{f}-\mathrm{l}$ and Supplementary Fig. $4 a-d)$. Consistent with the changes observed in 3-week-old juvenile bone, substantially reduced numbers of Runx $2^{+}$preosteoprogenitors and Osterix $^{+}$osteoprogenitors were also detected in 10 -week-old adult $Z e b 1^{\triangle E C}$ tibia (Supplementary Fig. 4e, f). Similarly, no considerable change in the TRAP ${ }^{+}$ osteoclasts numbers was observed in the tibia of 10-week-old $Z e b 1^{\triangle E C}$ mice compared with Zeb1 $1^{\triangle E C}$ mice (Supplementary Fig. 4g, h).
ZEB1 depletion represses Dll4/Notch signaling in bone ECs. To investigate the potential mechanisms by which endothelial ZEB1 regulates bone angiogenesis and osteogenesis, bone ECs of $Z e b 1^{W T}$ and $Z e b 1^{\triangle E C}$ mice were freshly isolated and subjected to RT-qPCR screening analysis. As shown, transcript levels of Dll4, Notch1, Notch2, Notch3, Notch4, Hes1, Hes5, Hey1, Heyl, and $E f n b 2$ were markedly reduced in $Z e b 1^{\Delta E C}$ mice, suggesting that the Notch signaling was downregulated in the $Z e b 1^{\Delta \mathrm{EC}}$ bone ECs (Fig. 4a). Intriguingly, Zeb2 transcript levels were also remarkably decreased in Zeb1 $1^{\triangle E C}$ bone ECs (Fig. 4a), suggesting that ZEB1 excision in bone ECs does not induce a compensatory increase in Zeb2 expression but markedly reduces its expression. Further, immunofluorescence analysis revealed that expression levels of Dll4 and Notch1 in $\mathrm{EMCN}^{+}$bone ECs were markedly decreased in the tibia of $Z e b 1^{\triangle E C}$ and $Z e b 1^{i \triangle E C}$ mice relative to their corresponding littermate controls (Fig. 4b, e). To assess whether ZEB1 controls Notch activity in an EC-autonomous manner, we performed a RT-qPCR assay on the in vitro cultured bone ECs that were isolated from Zeb1 $1^{f l f l}$ tibia and infected with adeno$\beta \mathrm{Gal}$ or adeno-Cre (to generate control and ZEB1-deleted cells, respectively). The results showed a significant reduction in transcript levels of Zeb1, Zeb2, and key components of Dll4/ Notch pathway in ZEB1-deleted bone ECs relative to control cells (Fig. 4f, g). Protein levels of Dll4, Notch1, and the intracellular domain of Notch1, NICD1 (which is the active form of Notch1), were strongly decreased in ZEB1-deleted bone ECs compared with control cells, as assessed by immunoblot and immunofluorescence analyses (Fig. 4h, i).

Similar to a well-established zinc-finger transcription factor Snail125-27, ZEB1 acts as a transcriptional repressor/activator by directly binding to E-box elements (consensus sequence, CANNTG), which are located within the promoter regions of target genes ${ }^{28,29}$. In this regard, initial searches identified multiple conserved E-box elements within the proximal regions of the murine Dll4 and Notch1 promoters (Fig. 5a). Indeed, ZEB1deleted bone ECs markedly decreased luciferase activity of wildtype (WT) Dll4 and Notch1 reporter constructs, without affecting luciferase activity of mutant (MUT) Dll4 and Notch1 reporter constructs harboring successive point mutations in all E-boxes (Fig. 5b). In control bone ECs, luciferase activity of MUT Dll4 and Notch1 reporter constructs was significantly lower than corresponding WT reporter constructs, confirming that ZEB1 functions as a transcription activator of Dll4 and Notch1 genes (Fig. 5b). Conversely, exogenous ZEB1-expressing bone ECs remarkably increased luciferase activity of WT Dll4 and Notch1 reporter constructs, wereas it failed to affect luciferase activity of MUT Dll4 and Notch1 reporter constructs (Fig. 5c). After chromatin immunoprecipitation (ChIP) of endogenous ZEB1 in bone ECs followed by PCR of the proximal and distal regions of the murine Dll4 and Notch1 promoters, ZEB1-ChIPs were found 
a

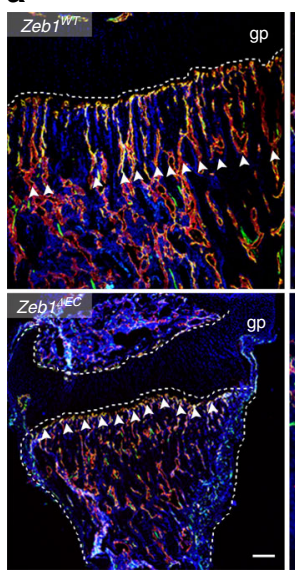

e

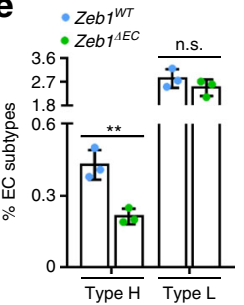

j

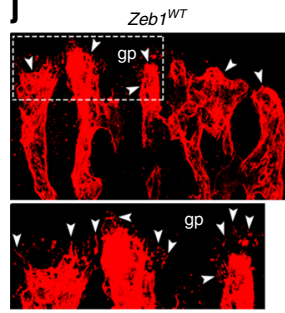

CD31/EMCN/DAPI

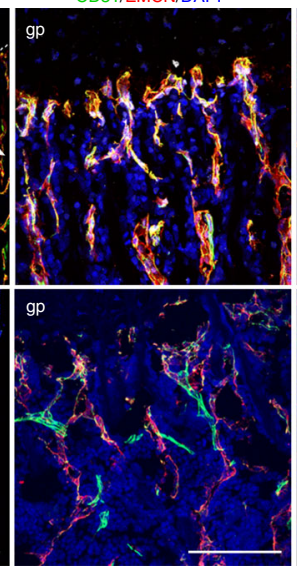

f

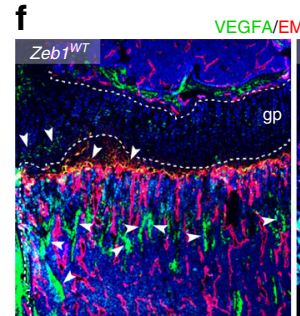

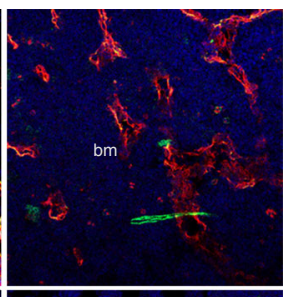
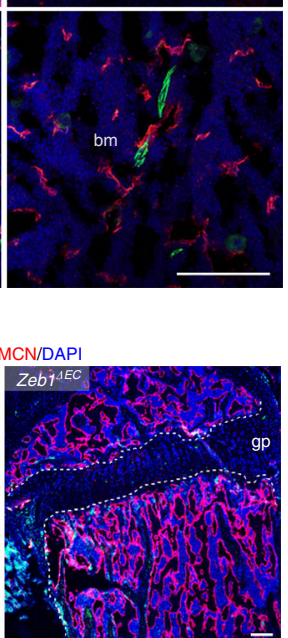

$\mathbf{k}$

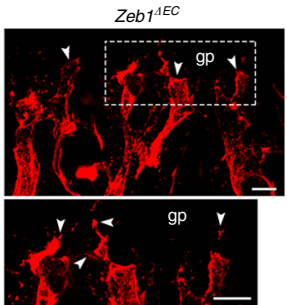

b
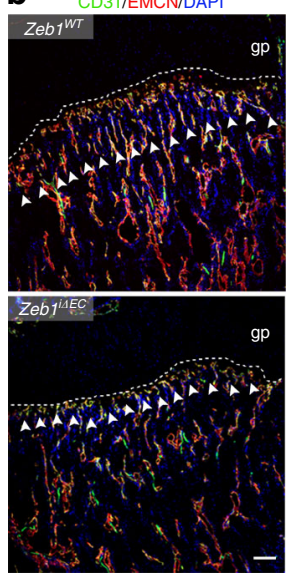

C
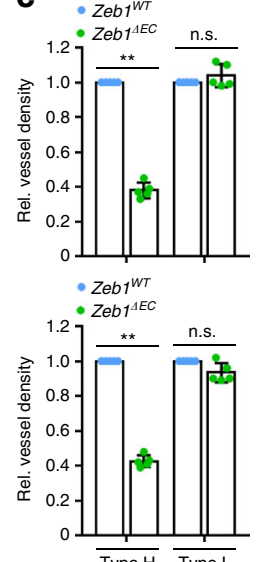

$\overline{\text { Type } H} \frac{}{\text { Type L }}$ d

Gated on DAPl ${ }^{-}$ Ter119-CD45- cells Zeb1 ${ }^{\text {WT }}$ Type H
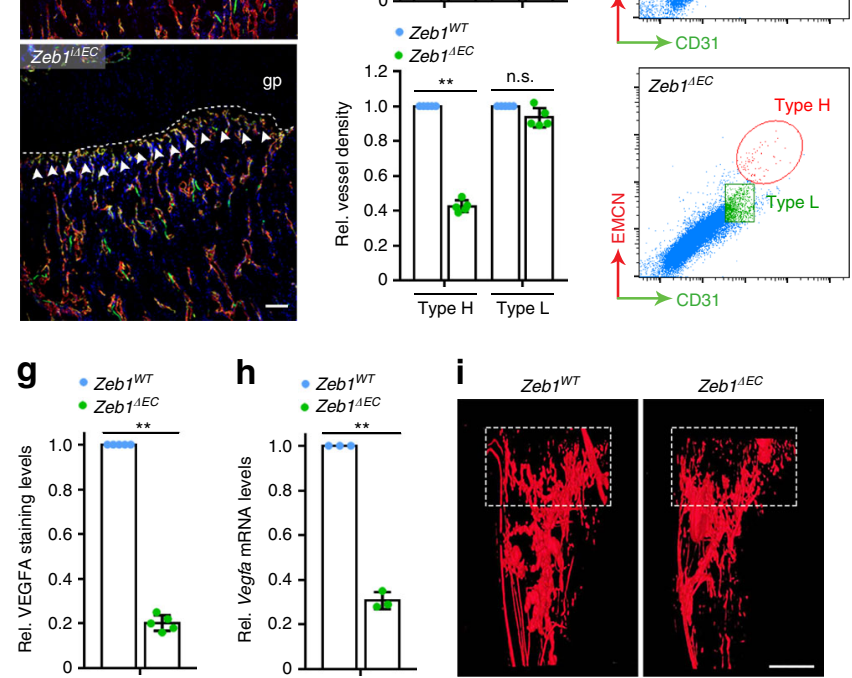

$Z e b 1^{A E C}$

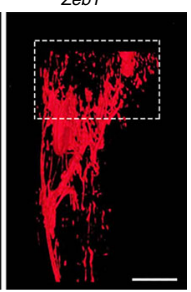

Fig. 2 EC-specific deletion of ZEB1 reduces bone angiogenesis. a, b Representative confocal images of CD31/EMCN immunostaining in tibia of juvenile 3week-old Zeb7 ${ }^{\triangle E C}$ mice $(\mathbf{a} ; n=5)$ and Zebi ${ }^{i \Delta E C}$ mice $(\mathbf{b} ; n=5$, each), and their corresponding littermate controls $(n=5$, each). Magnified images of type $\mathrm{H}$ and type $L$ vessels are shown in middle and right panels (a), respectively. Scale bar, $100 \mu \mathrm{m}$. Arrowhead denotes interface between type $H$ and $L$ vessels. $\mathbf{c}$ Quantification of type $\mathrm{H}$ and type $\mathrm{L}$ vessel densities in tibia as described in $\mathbf{a}$ and $\mathbf{b}$ ( $n=5$ independent experiments). d FACS plots of CD31 and EMCN double-stained single-cell suspensions from 3-week-old Zeb7 ${ }^{W T}$ and $Z e b 7^{\Delta E C}$ tibia $\left(n=5\right.$, each). e Quantification of type $\mathrm{H}$ and type $\mathrm{L}$ bone ECs in $\mathrm{LIN}{ }^{-}$(i.e., DAPI-Ter119-CD45-) cells as shown in $\mathbf{d}\left(n=5\right.$ independent experiments). f Confocal images of 3-week-old Zeb7WT and Zeb1 ${ }^{\Delta E C}$ tibia ( $n=5$, each) immunostained with VEGFA and EMCN. Scale bar, $100 \mu \mathrm{m}$. Arrowhead marks VEGFA positively staining in endothelial column/arches, surrounding perivascular cells, and mature/hypertrophic chondrocytes. $\mathbf{g}$ Quantification of VEGFA staining levels in tibia as shown in $\mathbf{f}(n=5$ independent experiments). h RT-qPCR analysis of Vegfa transcript in FACS-sorted bone ECs of 3-week-old Zeb7WT and Zeb7 ${ }^{\Delta E C}$ mice ( $n=3$ independent experiments). $\mathbf{i}$ Representative angiographic images of vascular organization of 3-week-old Zeb7WT and Zeb7 ${ }^{\triangle E C}$ tibia $(n=5$, each). Dashed boxed sections denote vessels near metaphysis. Scale bar, $0.5 \mathrm{~mm}$. j Confocal images of EMCN immunostained distal columns and arches next to gp in 3-week-old Zeb7WT and Zeb1 ${ }^{2 E C}$ tibia $(n=5$, each). Arrowhead denotes filopodia extension. Magnified areas of dashed boxed sections are shown in bottom panels. Scale bar, $20 \mu \mathrm{m}$. $\mathbf{k}$ Quantification of filopodia numbers in tibia as shown in $\mathbf{j}$ ( $n=5$ independent experiments). All data are represented as mean \pm SD. ${ }^{\star \star} P<0.01 ;$ NS not significant. Differences are tested using unpaired two-tailed Student's $t$-test. The source data are provided as a Source Data file.

to be enriched in the proximal, but not distal, regions of both promoters, suggesting that ZEB1 activated Dll4 and Notch1 transcription by specifically binding to promoter proximal regions (Fig. 5d, e). The ability of zinc-finger transcription factors (e.g., Snail) to transactivate target genes has been linked to the recruitment of histone-modifying cofactors to the promoters of target genes, which can specifically enhance histone acetylation but not methylation on the promoters ${ }^{25,30}$. ChIP-qPCR analysis showed that ZEB1-deleted bone ECs significantly reduced histone acetylation, including histone $\mathrm{H} 3$ lysine 4 acetylation (H3K4Ac), H3K14Ac, and H3K18Ac, on the promoters of Dll4 and Notch1, without affecting recruitment of histone $\mathrm{H} 3$ lysine 27 trimethylation (H3K27me3), a reported repressive histone marker 25,31
(Fig. 5f). To uncover the cofactors that are responsible for histone acetylation on the promoters of Dll4 and Notch1, we performed co-IPexperiments in bone ECs. The results demonstrated that ZEB1 was associated with histone acetyltransferases (HATs), CREB-binding protein (CBP)/p300 in bone ECs (Fig. 5g). Sequential ChIP analyses revealed that ZEB1 and CBP cooccupied Dll4 and Notch1 promoters in bone ECs (Fig. 5h). A promoter luciferase reporter assay was then performed in 293T cells that were transiently transfected with HA-ZEB1, HA-p300, or HA-CBP alone, or ZEB1 in combination with p300 or CBP (Fig. 5i). The results showed that overexpression of ZEB1, p300, or CBP alone increased Dll4 (Notch1) promoter activity by $88 \%(75 \%), 20 \%(16 \%)$, or $23 \%(15 \%)$, respectively, whereas 


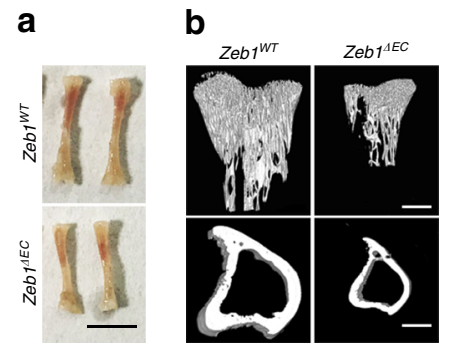

d
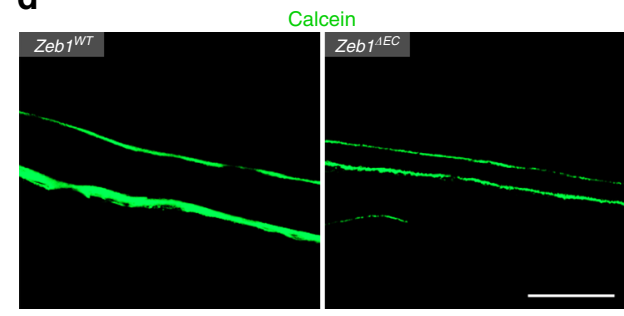

h
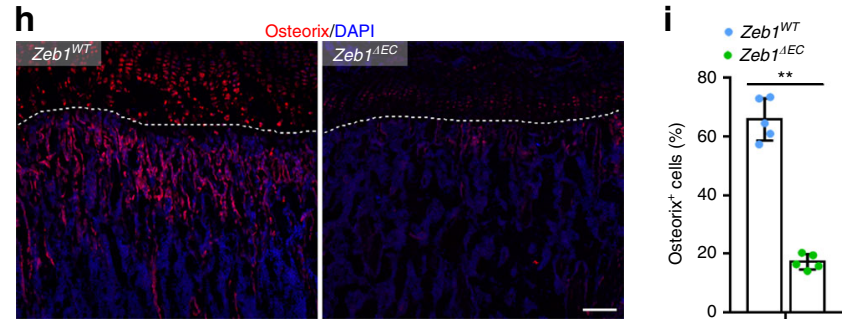

e
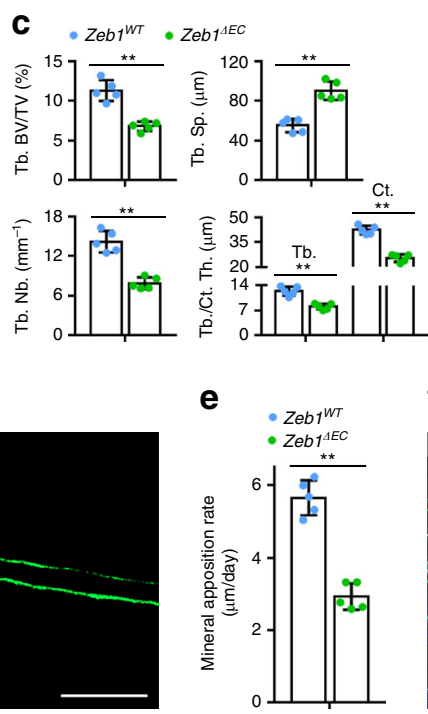

f
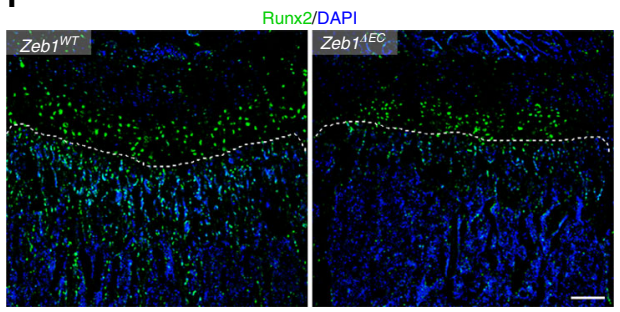

$9 \cdot Z e b 1^{w T}$

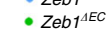
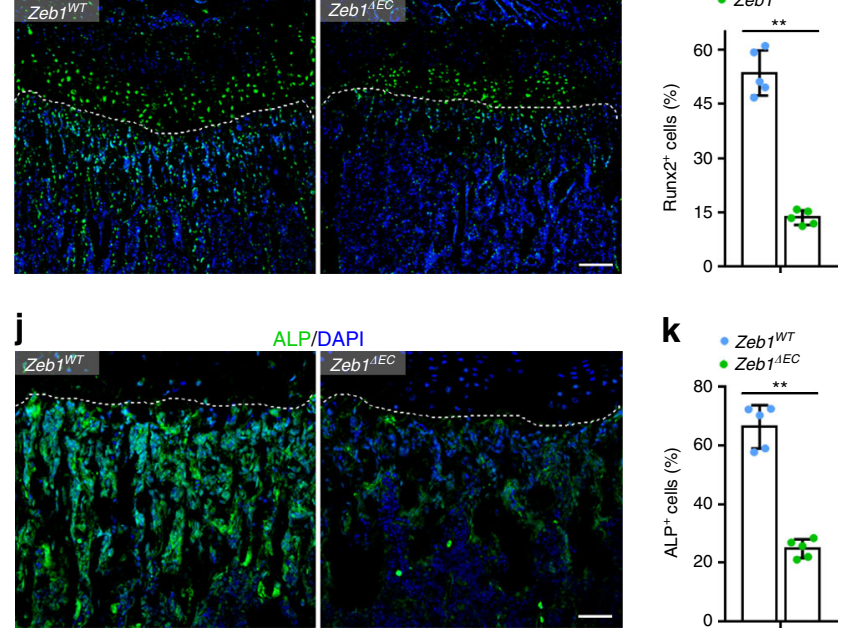

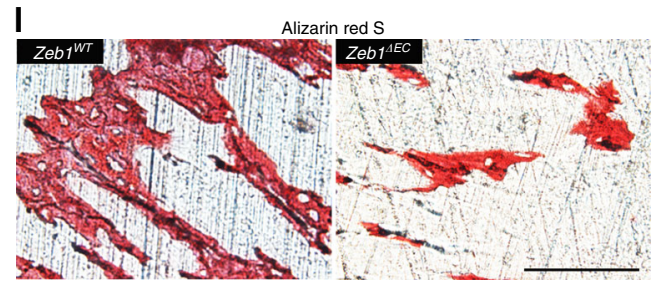

Fig. 3 EC-specific deletion of ZEB1 impairs angiogenesis-dependent bone formation. a Representative images of freshly dissected tibia of 3-week-old Zebi ${ }^{W T}$ and Zeb1 ${ }^{\triangle E C}$ mice $(n=5$, each). Scale bar, $50 \mathrm{~mm}$. b Representative micro-CT images of trabecular bone (top panels) and cortical bone (bottom panels) of 3-week-old Zeb7WT and Zeb7 $7^{\Delta E C}$ mice ( $n=5$, each). Scale bar, $0.2 \mathrm{~mm}$. c Quantification of Tb. BV/TV trabecular separation (Tb. Sp.), Tb. Nb. trabecular thickness (Tb. Th.), cortical thickness (Ct. Th.) in the tibia as shown in $\mathbf{b}$ ( $n=5$ independent experiments). $\mathbf{d}$ Representative images of calcein double labeling of Zeb7WT and Zeb7 ${ }^{\Delta E C}$ tibia $(n=5$, each). Scale bar, $100 \mu \mathrm{m}$. e Quantification of dynamic bone formation in tibia as shown in d ( $n=5$ independent experiments). $\mathbf{f}, \mathbf{h}, \mathbf{j}$ Representative images of 3-week-old Zeb1WT and Zeb1 ${ }^{\mathrm{WEC}}$ tibia ( $n=5$, each) immunostained with Runx2 (f), Osterix $(\mathbf{h})$, and ALP (j). Scale bar, $100 \mu \mathrm{m} . \mathbf{g}, \mathbf{i}, \mathbf{k}$ Quantification of Runx $2^{+}(\mathbf{g})$, Osterix ${ }^{+}(\mathbf{i})$, and $\operatorname{ALP}^{+}(\mathbf{k})$ cells in the tibia as shown in $\mathbf{f}, \mathbf{h}, \mathbf{j}(n=5$ independent experiments). I Representative images for Alizarin red S staining of 3-week-old Zeb1 ${ }^{W T}$ and Zeb1 ${ }^{\Delta E C}$ tibia $(n=5$, each). Scale bar, $100 \mu$ m. All data are represented as mean $\pm S D$. ${ }^{\star \star} P<0.01$; NS not significant. Differences are tested using unpaired two-tailed Student's $t$-test. The source data are provided as a Source Data file.

overexpression of ZEB1 in combination with p300 or CBP resulted in a 6.0 (4.1)-fold or 7.0 (5.4)-fold increase in Dll4 (Notch1) promoter activity, respectively (Fig. 5j). These findings suggest that ZEB1 interacts with $\mathrm{CBP} / \mathrm{p} 300$ in bone ECs and they co-occupy the promoters of Dll4 and Notch1 to enhance histone acetylation on the promoters, thus inducing transcriptional activation of Dll4 and Notch1.

Treatment with r.Dll4 protein restores bone formation. Given these results, we next sought to determine the degree to which Dll4 serves as a ZEB1 target substrate required for angiogenesis and bone formation. Treatment of in vitro cultured ZEB1-deleted bone ECs with recombinant Dll4 (r.Dll4) protein remarkably restored expressions of key components of Dll4/Notch pathway such as Dll4, Notch1, Hes1, Hey1, and Efnb2, as assessed by RTqPCR analysis (Supplementary Fig. 5a). Importantly, the impaired expression of NICD1 in ZEB1-deleted bone ECs was also fully recovered following r.Dll4 treatment, as assessed by immunofluorescence and immunoblot analyses (Fig. 6a, b), suggesting that ZEB1-deleted bone ECs with impaired Notch activity were responsive to Dll4 stimulation. Our findings are consistent with previous reports demonstrating that treatment of muscle satellite cells and ECs with r.Dll4 protein robustly stimulates Notch activity $^{32,33}$. Daily injection of $Z e b 1^{W T}$ mice with $1 \mu \mathrm{g} / \mathrm{g}$ r.Dll4 protein for 2 consecutive weeks (starting from P4) before analysis at P21 markedly increased protein expressions of Dll4 and Notch1 in the tibia (Supplementary Fig. 5b), but had no obvious impact on body weight (Supplementary Fig. 5c), bone angiogenesis (Fig. 6c, d), or osteogenesis (Fig. 6e-h). In sharp contrast, administration of $Z e b 1^{\triangle E C}$ mice with r.Dll4 protein remarkably restored body weight (Supplementary Fig. 5c), enhanced type $\mathrm{H}$ vessel formation and VEGFA expression in the tibia (Fig. 6c, d), 
a

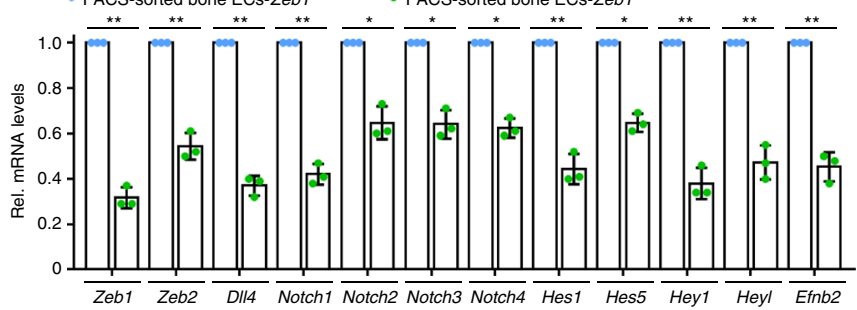

d

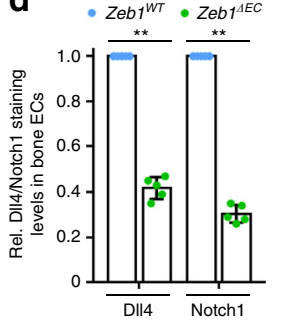

e

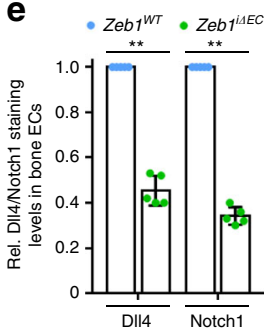

b
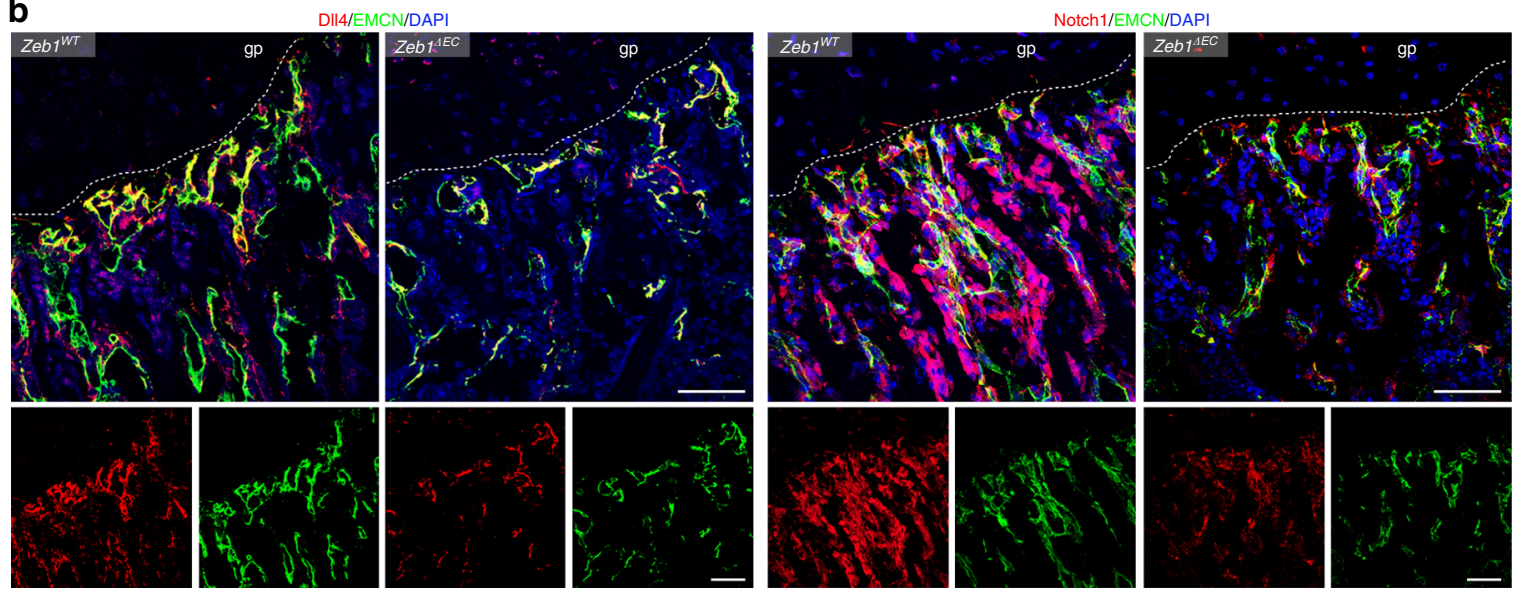

C

DII4/EMCN/DAP
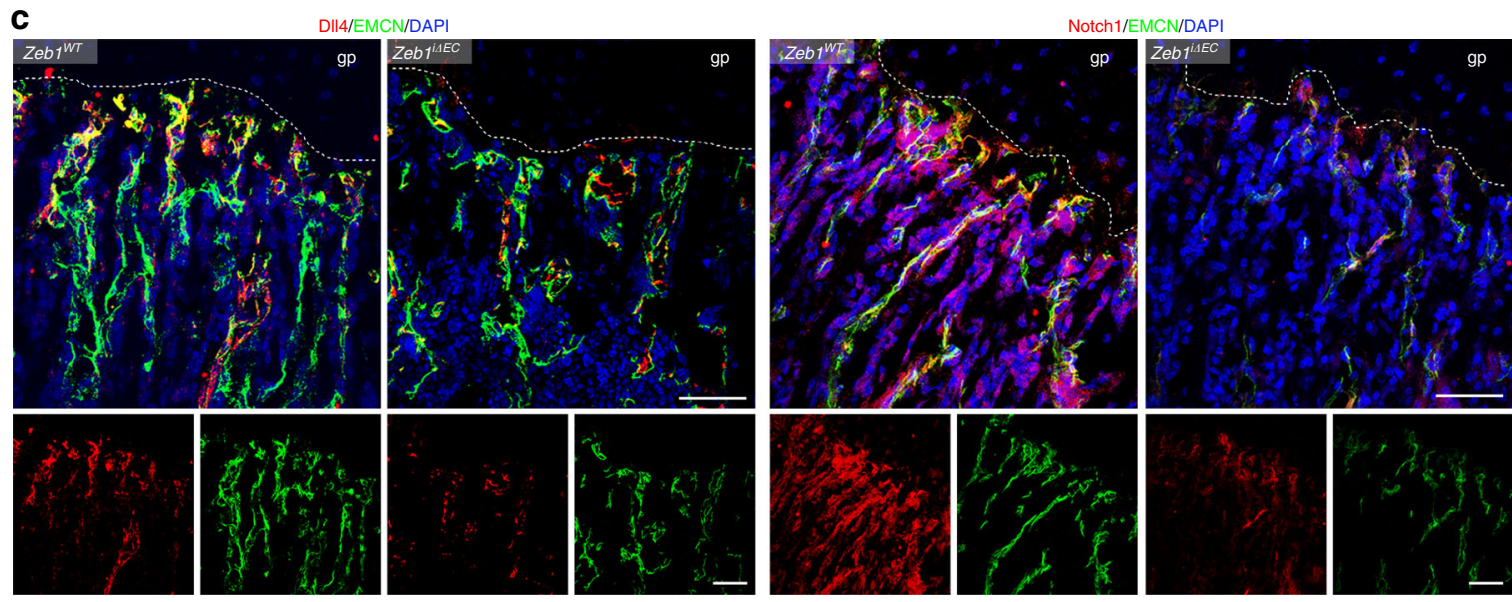

f

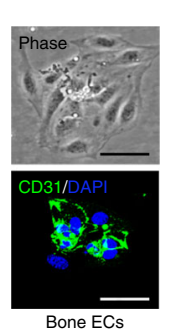

9 Cultured bone ECs (Ad- $\beta$ Gal)
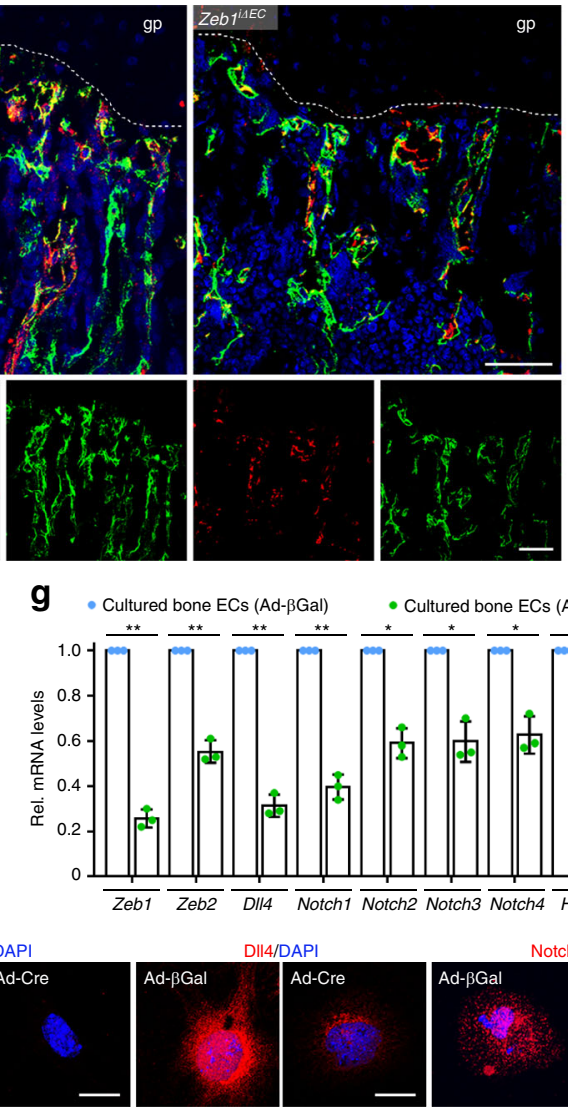

$\mathbf{h}_{\mathrm{A} \text { }}$

$\underset{\text { Ad-Cre }}{\text { Ad- }}++\left.\right|_{\text {bone ECs }} ^{\text {Cultured }}$
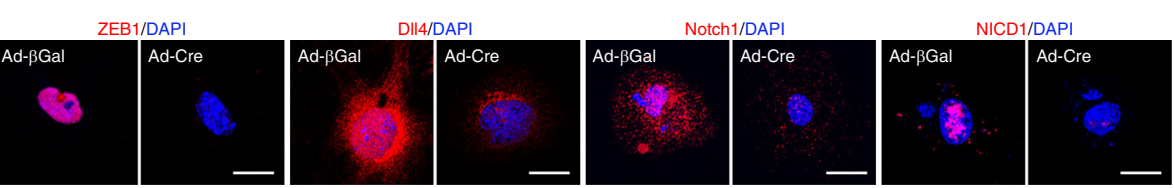

improved bone formation (Fig. 6e, f), and normalized the numbers of Run $\times 2^{+}$pre-osteoprogenitors, Osterix ${ }^{+}$osteoprogenitors, and $\mathrm{ALP}^{+}$mature osteoblasts (Fig. 6g, h). Recently, angiocrine factors secreted by vascular niche have been reported to play critical roles in promoting organ development, tumor growth, and tissue regeneration ${ }^{34,35}$. We therefore sought to identify potential pro- osteogenic factors that are actively involved in regulation of endothelial Notch-dependent osteogenesis. As shown, Tgfb1, Tgfb2, Tgfb3, Bmp2, Bmp4, Pgf (encoding PLGF), Fgf1, and Nog (encoding Noggin) transcripts were dramatically reduced in FACS-sorted bone ECs of Zeb1 ${ }^{\Delta E C}$ mice, among which Tgfbl, Tgfb2, Bmp2, Bmp4, Fgfl, and Nog transcripts were markedly 
Fig. 4 Endothelial ZEB1 inactivation downregulates Notch activity. a RT-qPCR analysis of Zeb1, Zeb2, DIl4, Notch1, Notch2, Notch3, Notch4, Hes1, Hes5, Hey1, Heyl, and Efnb2 in FACS-sorted bone ECs of 3-week-old Zeb1WT and Zeb1 ${ }^{\Delta E C}$ mice ( $n=3$ independent experiments). b, c Representative confocal

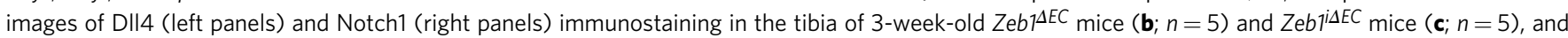
their corresponding littermate controls $(n=5$, each). Scale bar, $60 \mu \mathrm{m}$. d Quantification of DII4 (left panels) and Notch1 (right panels) staining levels in $\mathrm{EMCN}^{+}$bone ECs of 3-week-old Zeb7 ${ }^{W T}$ and Zeb7 ${ }^{\triangle E C}$ mice as described in $\mathbf{b}$ ( $n=5$ independent experiments). e Quantification of DII4 (left panels) and Notch1 (right panels) staining levels in EMCN ${ }^{+}$bone ECs of 3-week-old Zebi ${ }^{W T}$ and Zeb7i ${ }^{i \Delta C}$ mice as described in $\mathbf{c}(n=5$ independent experiments). $\mathbf{f}$ Representative phase contrast (top panel) and confocal (CD31 immunostaining; bottom panel) images of magnetic activated cell sorting (MACS)-sorted bone ECs. Scale bar, $50 \mu \mathrm{m}$. $\mathbf{g}$ RT-qPCR analysis of Zeb1 and the indicated Notch pathway components in the in vitro cultured control (i.e., adeno- $\beta$ Galinfected) and ZEB1-deleted (adeno-Cre-infected) bone ECs ( $n=3$ independent experiments). $\mathbf{h}$ Immunoblot analysis of ZEB1, DII4, Notch1, and NICD1 in control and ZEB1-deleted bone ECs as described in $\mathbf{g}$. Representative blots as shown are from three independent experiments. i Representative confocal images of control and ZEB1-deleted bone ECs with immunostaining of ZEB1, DII4, Notch1, and NICD1. Scale bar, $20 \mu \mathrm{m}$. Images as shown are from three independent experiments. All data are represented as mean \pm SD. ${ }^{\star}{ }^{\star} P<0.01,{ }^{\star} P<0.05$. Differences are tested using one-way ANOVA with Tukey's post hoc test $(\mathbf{a}, \mathbf{g})$ and unpaired two-tailed Student's $t$-test $(\mathbf{d}, \mathbf{e})$. The source data are provided as a Source Data file. Unprocessed original scans of blots are shown in Source Data file.

restored in bone ECs of $Z e b 1^{\Delta E C}$ mice that were administrated with r.Dll4 protein (Supplementary Fig. 5d). Our data demonstrate that Dll4/Notch1 signaling pathway is actively involved in the regulation of endothelial ZEB1 on bone angiogenesis and osteogenesis.

Zeb1 gene delivery alleviates OVX-induced bone loss. Evidence to date indicates that type $\mathrm{H}$ vessel numbers are significantly lower in the bone of osteoporotic humans and mice ${ }^{4,6-8}$. Notably, ZEB1 expression was significantly reduced in bone ECs of osteoporotic patients relative to normal controls, a finding accompanied by substantially decreased densities of total vessels and type $\mathrm{H}$ vessels (Fig. 7a, b). Based on these findings, we decided to determine whether pharmacological administration of exogenously expressed ZEB1 could ameliorate osteoporosis using a DNA-loaded cationic liposome vehicle ${ }^{36-38}$ and an OVX mouse model $^{4,8}$.

To directly assess the efficiency and specificity of cationic liposome-delivered Zeb1 gene in vivo, we generated a pcDNA3.1 $+\mathrm{C}$-eGFP-ZEB1 vector (designed ZEB1-GFP) that can express recombinant enhanced greenfluorescent protein (eGFP)-fused ZEB1 protein in the target cells. Next, pcDNA3.1+C-eGFP (designed Vector-GFP) or ZEB1-GFP was mixed with cationic protamine at different $\mathrm{N}: \mathrm{P}$ ratios (the molar ratio of nitrogen in protamine to phosphate in DNA vector) to form the nano-sized DNA/protamine complex (designed Protamine-Vector-GFP and Protamine-ZEB1-GFP). DNA packing efficiency was examined by agarose gel electrophoresis. The results showed that protamine can completely condense Vector-GFP and ZEB1-GFP at the N:P ratio of $3: 1$ and 4:1, respectively (Supplementary Fig. 6a). Protamine-Vector-GFP or Protamine-ZEB1-GFP was subsequently mixed with cationic liposomes at $60 \mathrm{nmol} \mathrm{lipid} / \mu \mathrm{g}$ DNA to generate Lipo.-Vector-GFP and Lipo-ZEB1-GFP, respectively. Agarose gel electrophoresis analysis revealed that Vector-GFP and ZEB1-GFP were completely encapsulated in the cationic liposomes under these conditions (Supplementary Fig. 6b). The final Lipo-Vector-GFP had a particle size of $151.2 \pm 5.7 \mathrm{~nm}$ (mean $\pm \mathrm{SD}$ ), a zeta potential of $+24.9 \pm 1.6 \mathrm{mv}$, and a polymer dispersity index of $0.286 \pm 0.017$, respectively, whereas the final Lipo.-ZEB1-GFP had $147.5 \pm 4.9 \mathrm{~nm}, 23.8 \pm 2.1$ $\mathrm{mv}$, and $0.270 \pm 0.017$, respectively (Supplementary Fig. 6c), confirming largely comparable physicochemical characteristics of Lipo--Vector-GFP vs. Lipo-ZEB1-GFP. We sought to analyze pharmacokinetic properties of the DNA-packaged liposomes. To this end, Vector-GFP and ZEB1-GFP were encapsulated into rhodamine phycoerythrin (PE)-labeled liposomes, and the DNApackaged liposomes (designed Rho.-Lipo.-Vector-GFP and Rho.Lipo.-ZEB1-GFP, respectively) were intravenously (i.v.) injected into rats. The blood samples collected at different times post treatment were centrifuged and the supernatant plasma was collected for fluorescence intensity measurement. As shown, the fluorescence intensity-time curves of Rho.-Lipo.-Vector-GFP and Rho.-Lipo.-ZEB1-GFP were largely comparable (Supplementary Fig. 6d). Pharmacokinetic parameters such as $t_{1 / 2}$ (elimination half-life), $C_{\max }$ (maximal plasma concentration), $\mathrm{AUC}_{0-\infty}$ (area under the plasma concentration-time curve), and MRT (mean residence time) of Rho.-Lipo.-Vector-GFP and Rho.-Lipo--ZEB1GFP were also comparable (Supplementary Fig. 6e). We next sought to analyze biodistribution of the DNA-packaged liposomes. For this purpose, ZEB1-GFP was packaged into 1,1'dioctadecyl-3,3,3',3'-tetramethylindotricarbocyanine iodide (DiR)-labeled liposomes and the DNA-packaged liposomes (designed DiR-Lipo-ZEB1-GFP) were i.v. injected into adult mice; the kidneys, spleen, lungs, brain, heart, liver, and hind limbs were dissected $24 \mathrm{~h}$ post injection and subjected to ex vivo biophotonic imaging analysis. As shown, the DiR signal was readily detected in the hind limbs, liver, spleen, lung, and kidneys, whereas the signal was hardly undetectable in the brain and heart (Supplementary Fig. 6f, g), validating efficient distribution of DiR-Lipo-ZEB1-GFP to the skeletal and non-skeletal organs.

To generate OVX and sham-operation (Sham) mouse models, the surgery was performed on 8-week-old female mice and the mice at day 3 post surgery were i.v. injected with $4 \mu \mathrm{g}$ Lipo.Vector-GFP or Lipo.-ZEB1-GFP twice every week for consecutive 6 weeks. As predicted, uterine weight of OVX mice was reduced by $75 \%$ compared with Sham mice, whereas uterine weight of OVX mice treated with Lipo.-Vector-GFP or Lipo.-ZEB1-GFP was largely comparable (Supplementary Fig. 7a, b). To examine expressions of liposome-delivered Vector-GFP and ZEB1-GFP in target cells, the tibia and non-skeletal tissues were dissected for immunofluorescence analysis. The results revealed that liposomedelivered Vector-GFP and ZEB1-GFP were predominantly expressed in the metaphysis of the tibia (Fig. 7c) with remarkably lower expressions in the diaphysis of the tibia and non-skeletal tissues such as the lung, liver, and spleen (Supplementary Fig. 7c). Notably, a large proportion of $\mathrm{GFP}^{+}$cells was identified as $\mathrm{EMCN}^{+}$bone ECs, indicating that bone ECs in the metaphysis of the tibia are the major target cells of Lipo.-Vector-GFP and Lipo.ZEB1-GFP (Fig. 7c). However, a small proportion of $\mathrm{GFP}^{+}$cells can be identified as $\mathrm{EMCN}^{-}$perivascular cells that may also respond to the liposome treatment (Fig. 7c). Furthermore, Vector-GFP recombinant protein was localized in cytoplasmic fraction of metaphyseal cells (in green; Fig. 7c, left and middle panels), whereas ZEB1-GFP recombinant protein was localized in the nucleus of metaphyseal cells (in yellow; Fig. 7c, right panels). In OVX mice treated with Lipo-ZEB1-GFP, the positivity of endogenous $\mathrm{ZEB} 1^{+}$bone ECs (i.e., the ratio of red/white: white) and exogenous $\mathrm{ZEB1}^{+}$bone ECs (i.e., the ratio of yellow/white: 

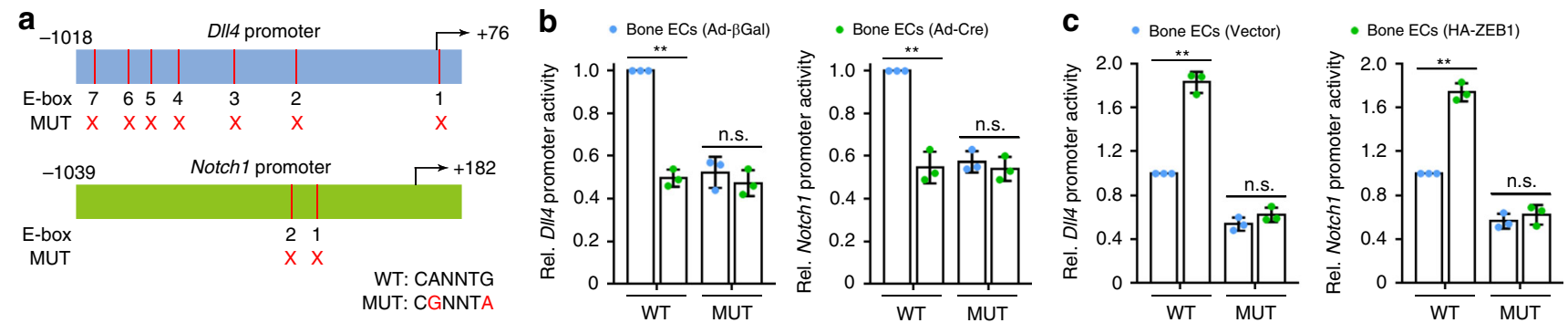

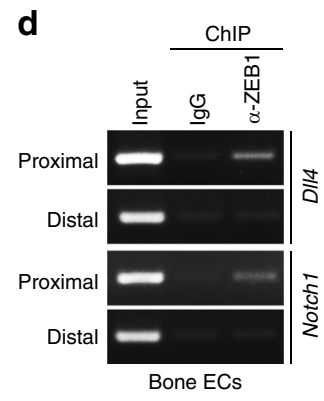

e

g

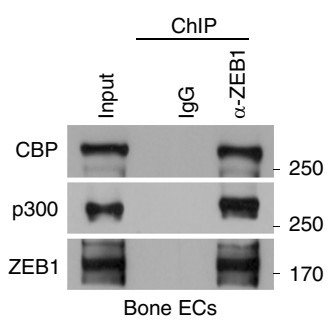

e $\quad \cdot \lg 6 \cdot 02 E B$

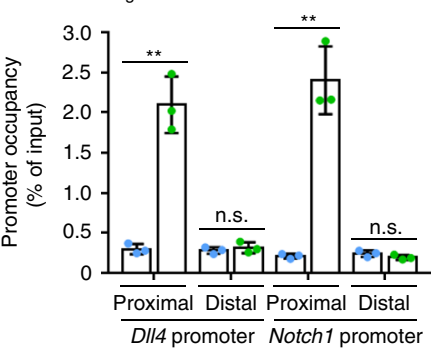

h

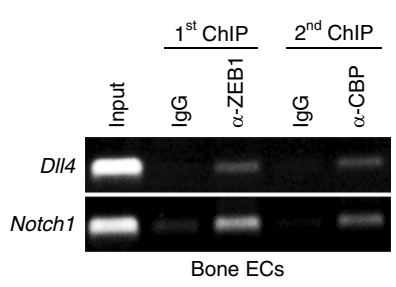

f
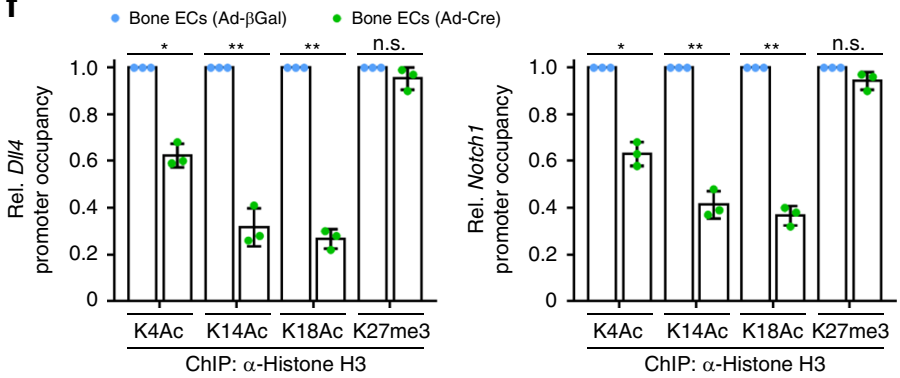

i

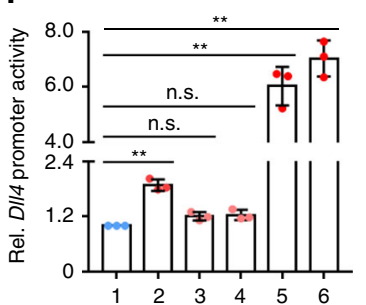

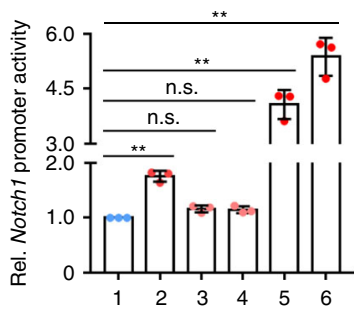

j

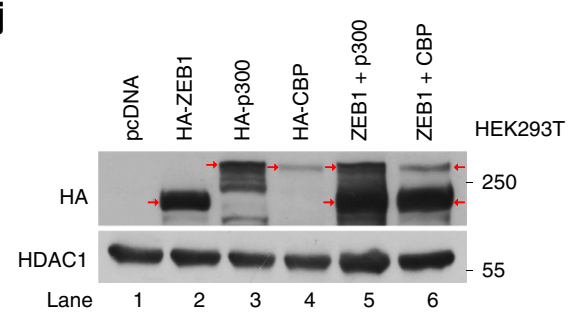

Fig. 5 ZEB1 depletion epigenetically reduces DII4 and Notch1 expressions in bone ECs. a Schematic representation of reporter constructs of wild-type (WT) and mutated (MUT) murine DII4 and Notch1 promoters. b Luciferase reporter analysis of cultured control and ZEB1-deleted bone ECs that were electroporated with WT or MUT DII4 and Notch1 promoter reporter constructs ( $n=3$ independent experiments). $\mathbf{c}$ Luciferase reporter analysis of cultured bone ECs that were co-electroporated with WT or MUT DII4 and Notch1 promoter reporter constructs in combination with pcDNA3.1 or pcDNA3.1-HAZEB1 ( $n=3$ independent experiments). d, e ChIP-PCR analysis of interactions of ZEB1-DII4 promoter and ZEB1-Notch1 promoter within the proximal and distal regions in cultured bone ECs. Agarose gel images as shown are from three independent experiments (d) and promoter occupancy relative to corresponding input was quantified (e; $n=3$ independent experiments). prox. proximal, dist. distal. $\mathbf{f}$ ChIP-qPCR for analyzing the enrichments of H3K4Ac, H3K14Ac, H3K18Ac, and H3K27me3 on DII4 and Notch1 promoters in control and ZEB1-deleted bone ECs ( $n=3$ independent experiments). $\mathbf{g}$ Immunoprecipitation (IP) analysis of the physical interaction between ZEB1 and CBP or p300 in nuclear extracts of bone ECs. Images as shown are from three independent experiments. $\mathbf{h}$ Sequential ChIP-PCR analysis confirming the co-occupancy of ZEB1 and CBP or p300 on DII4 and Notch1 promoters in bone ECs. Images as shown are from three independent experiments. $\mathbf{i}, \mathbf{j}$ Luciferase reporter assays for analyzing DII4 and Notch1 promoter activity in HEK293T cells that were co-transfected with the indicated constructs (i). Immunoblot analysis confirming ectopic expression of HA tagged ZEB1, p300, and CBP in nuclear extracts of HEK293T cells (j). Images as shown are from three independent experiments. Arrow marks specific bands with the expected molecular weights, respectively. All data are represented as mean \pm SD. ${ }^{\star \star} P<0.01,{ }^{\star} P<0.05$; NS not significant. Differences are tested using one-way ANOVA with Tukey's post hoc test $(\mathbf{b}, \mathbf{c}, \mathbf{i})$ and unpaired two-tailed Student's t-test $(\mathbf{e}, \mathbf{f})$. The source data are provided as a Source Data file. Unprocessed original scans of blots are shown in Source Data file.

white) was $13.4 \%$ and $28.3 \%$, respectively, suggesting that exogenous recombinant ZEB1 protein was efficiently delivered to and expressed in $\mathrm{EMCN}^{+}$bone ECs (Fig. 7c, d). Endogenously expressed ZEB1 protein levels in bone ECs of OVX mice were decreased by $60 \%$ compared with Sham mice, and the levels of ZEB1 protein (including endogenously and exogenously expressed ZEB1) in bone ECs of OVX + Lipo.-ZEB1-GFP mice were restored to $90 \%$ those of Sham + Vector-GFP mice (Fig. 7c, d). Importantly, the impaired type $\mathrm{H}$ vessel formation in tibial metaphysis of OVX mice was efficiently recovered following Lipo.-ZEB1-GFP treatment (Fig. 7e, f). Accordingly, the diminished expressions of Dll4 and Notch1 proteins in $\mathrm{EMCN}^{+}$bone ECs of OVX mice were also considerably restored following Lipo.-ZEB1-GFP treatment (Fig. 7g, h). 


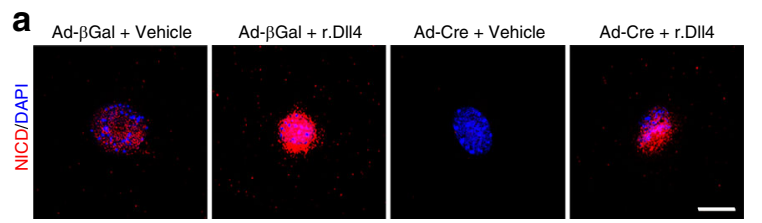

C

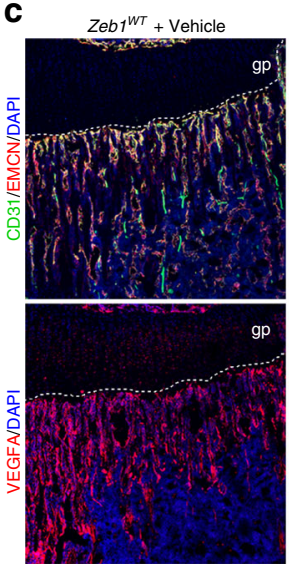

e
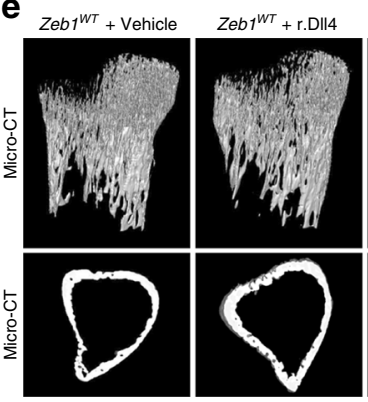

g

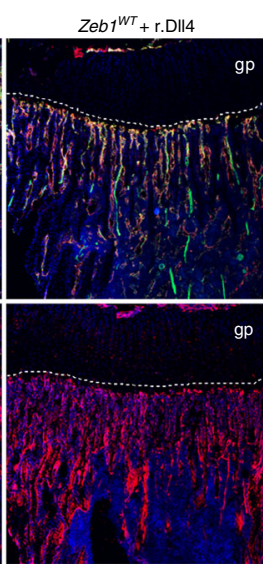

Zeb $1^{A E C}+$ Vehicle

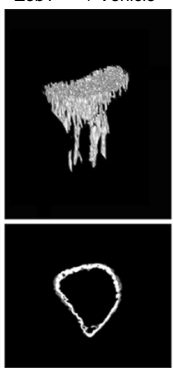

$Z e b 1^{W T}+$ r.DII4

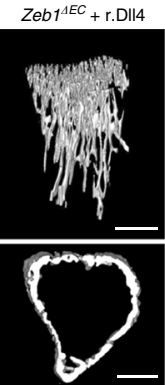

$Z e b 1^{4 E C}+$ Vehicle

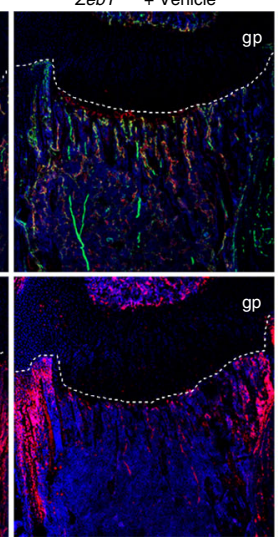

b

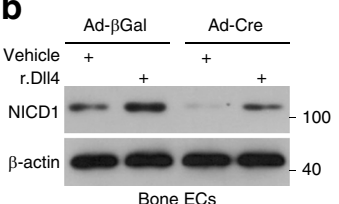

$Z e b 1^{\Delta E C}+$ r.DII4

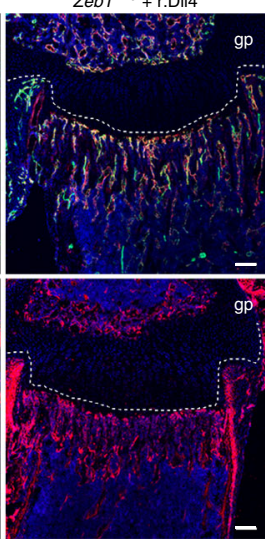

d
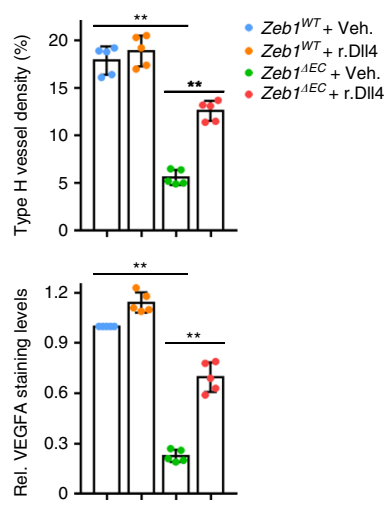
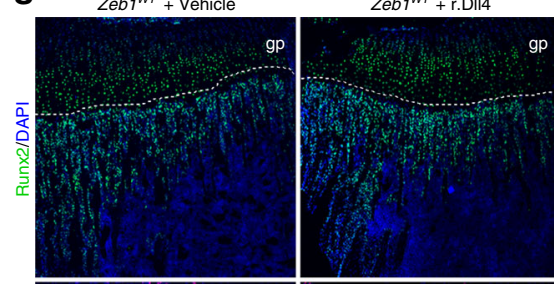

f
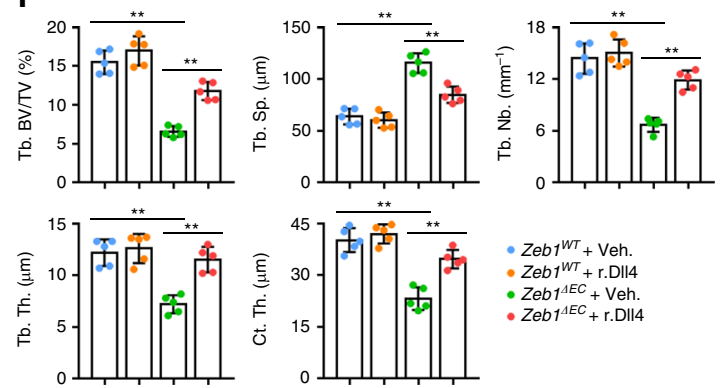

$Z e b 1^{W T}+$ Veh

- Zeb $1^{W T}+$ r.DII4

- Zeb1 $1^{A E C}+$ Veh.

$\mathbf{h}$
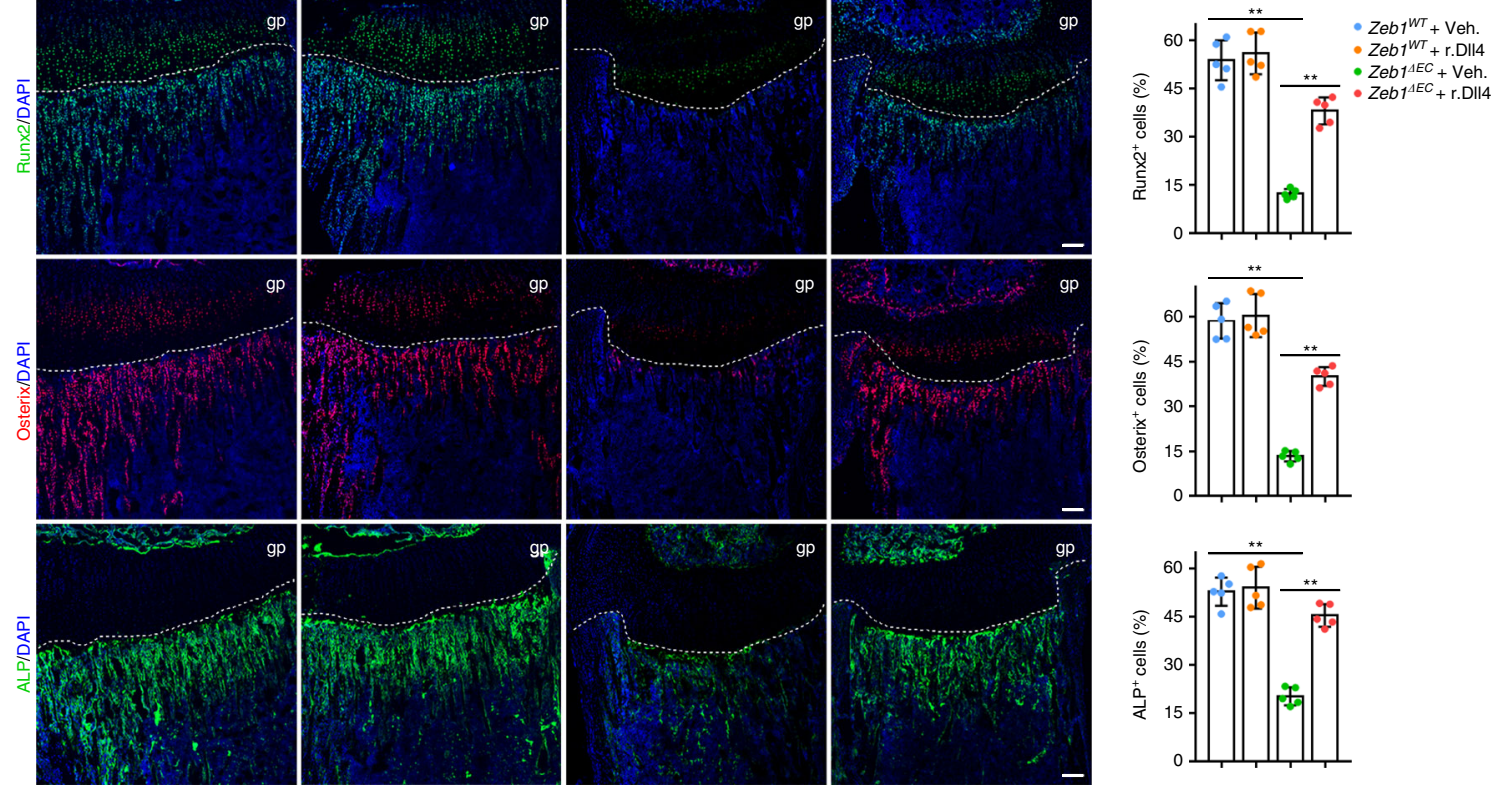

As predicted, micro-CT analysis demonstrated that tibia of OVX mice exhibited increased cross-sectional endosteal perimeters (might due to bone loss at the endosteum) in tandem with remarkably decreased Ct. Th., trabecular bone density, Tb. Nb., and Tb. Th. compared with Sham mice (Fig. 8a, b). Notably, administration of OVX mice with Lipo-ZEB1-GFP efficiently restored the impaired bone formation in the mice (Fig. 8a, b). Immunofluorescence analysis further revealed that tibia of OVX mice had significantly lower numbers of $\mathrm{Runx}^{+}$preosteoprogenitors and Osterix ${ }^{+}$osteoprogenitors than Sham mice (Fig. 8c, d), which is consistent with previous reports demonstrating that numbers of Runx $2^{+}$and Osterix ${ }^{+}$cells are strongly 
Fig. 6 Administration of Zeb1 ${ }^{\triangle E C}$ mice with r.DIl4 protein efficiently restores the impaired bone angiogenesis and osteogenesis. $\mathbf{a}$, $b$ Control and ZEB1deleted bone ECs seeded on vehicle- or r.Dll4-precoated chamber wells (or culture dishes) were subjected to NICD1 immunostaining (a) and immunoblotting (b) analyses, respectively. Representative confocal or immunoblot images as shown are from three independent experiments. Scale bar, $20 \mu \mathrm{m}$. c Representative confocal images of CD31/EMCN and VEGFA immunostaining in the tibia of Zeb7WT and Zeb1 ${ }^{\triangle E C}$ mice that were i.p. injected with $1 \mu \mathrm{g} / \mathrm{g}$ r.DIl4 protein at P4 for 2 consecutive weeks before analysis at P21 ( $n=5$, each). Scale bar, $100 \mu \mathrm{m}$. d Quantification of type H vessel density (top panel) and VEGFA staining levels (bottom) in the tibia as shown in c ( $n=5$ independent experiments). e Micro-CT analysis of the tibia showing improved bone formation in trabecular bone (top panels) and cortical bone (bottom panels) of Zeb7 ${ }^{\Delta \mathrm{EC}}$ mice treated with r.DII4 protein as described in $\mathbf{c}(n=5$, each). Scale bar, $0.2 \mathrm{~mm}$. $\mathbf{f}$ Quantification of bone architectures in the tibia as shown in $\mathbf{e}(n=5$ independent experiments). $\mathbf{g}$ Runx2, Osterix, and ALP immunostaining of the tibia as described in $\mathbf{c}\left(n=5\right.$, each). Scale bar, $100 \mu \mathrm{m}$. $\mathbf{h}$ Quantification of Runx2+, Osterix ${ }^{+}$, and ALP+ cells in the tibia as shown in $\mathbf{g}\left(n=5\right.$ independent experiments). All data are represented as mean \pm SD. ${ }^{\star \star} P<0.01$. Differences are tested using one-way ANOVA with Tukey's post hoc test $(\mathbf{d}, \mathbf{f}, \mathbf{h})$. The source data are provided as a Source Data file. Unprocessed original scans of blots are shown in Source Data file.

reduced in OVX bone ${ }^{39-41}$. As predicted, administration of OVX mice with Lipo-ZEB1-GFP efficiently normalized the numbers of $\mathrm{Runx}^{+}$pre-osteoprogenitors and Osterix ${ }^{+}$osteoprogenitors (Fig. 8c, d). To evaluate whether repeated treatments with Lipo.-ZEB1-GFP or Lipo.-Vector-GFP could induce any histological alterations in major organs, we harvested major organs such as the lung, spleen, heart, liver, and kidney of liposome-treated mice for histo-morphometric analysis. Importantly, the results showed that administration of Sham or OVX mice with Lipo.ZEB1-GFP or Lipo.-Vector-GFP for consecutive 6 weeks failed to induce any detectable histological alterations in the major organs, supporting the absence of Lipo-ZEB1-GFP- or Lipo.-VectorGFP-induced side toxicity (Supplementary Fig. 7d).

\section{Discussion}

In the present study, we unexpectedly found that EMT-associated transcription factor (EMT-TF) ZEB1 is predominantly expressed in type $\mathrm{H}$ vascular endothelium in human and murine bone. Type $\mathrm{H}$ vessels primarily reside in the bone marrow near the growth plate but are essentially undetectable in non-skeletal organs ${ }^{1-4}$. Recent studies have demonstrated that type $\mathrm{H}$ endothelium impacts bone architecture, bone formation, and the recruitment of perivascular osteoprogenitor ${ }^{1-4}$. EC-specific deletion of ZEB1 strongly reduces type $\mathrm{H}$ vessel growth and bone formation but does not affect vasculature growth or cause histological alterations in the non-skeletal organs. The considerably variable consequences of ZEB1 depletion in ECs are due to the highly differential expression of ZEB1 in skeletal type $\mathrm{H}$ endothelium vs. non-skeletal endothelium. The EMT activator ZEB1 is largely a transcriptional repressor. ZEB1 silencing in mesenchymal cancer cells restores transcription of $\mathrm{CDH} 1$ (encoding E-cadherin), a hallmark of EMT programs ${ }^{10,11}$. However, ZEB1 deletion in bone ECs does not affect $C D H 1$ transcription but indeed decreases histone acetylation on Dll4 and Notch1 promoters, thereby directly suppressing their transcriptional activity and downregulating endothelial Notch signaling. The evolutionarily conserved Notch signaling pathway plays critical roles in controlling multiple aspects of vascular development and EC function, ranging from proliferation, motility, and lumen formation to vessel stability and cell fate determination ${ }^{42,43}$. Notch signaling has been identified as a critical positive regulator of vascular growth in bone, which is highly distinct from the Notch-mediated suppression of vessel sprouting in non-skeletal organs and in malignant tumors ${ }^{44-46}$. A recent report has demonstrated that ZEB1 indirectly upregulates Notch signaling by reducing miR-200 expression in multiple tumor cell lines ${ }^{47}$. Here we demonstrate that ZEB1 interacts with HATs CBP/p300 and they co-occupy the promoters of Dll4 and Notch1 to enhance histone acetylation on the promoters, thus directly inducing transcriptional activation of Dll4 and Notch1 in bone ECs. Our results further demonstrate that ZEB1/Notch signaling axis plays an essential role in controlling type $\mathrm{H}$ bone vessel formation. Notch signaling has been reported to promote bone-specific angiogenesis and osteogenesis in both autocrine and paracrine manners ${ }^{2}$. Here we found that ZEB1/Notch signaling pathway controls recruitment/differentiation of perivascular osteoprogenitors and consequently promotes osteogenesis in the bone by regulating expression of multiple angiocrine factors such as TGF $\beta 1$, TGF $\beta 2$, BMP2, BMP4, FGF1, and Noggin. All these angiocrine factors are believed to actively participate in osteogenesis. Future work will address the mechanisms by which these angiocrine factors regulate differentiation and recruitment of osteoprogenitors in Zeb1 ${ }^{\Delta \mathrm{EC}}$ bone microenvironment.

In the present study, we observe that the impaired bone angiogenesis and osteogenesis in OVX mice are efficiently restored following systematic treatment with ZEB1-GFPpackaged cationic liposomes. Although an efficient distribution of DNA-packaged cationic liposomes to the bone and various non-skeletal organs is observed, the liposome-delivered plasmid DNAs are predominantly expressed in the metaphysis of the tibia (mostly in type $\mathrm{H}$ bone ECs) with remarkably lower expressions in the diaphysis of tibia and non-skeletal elements. One possible explanation for the considerable difference between the organ distribution of DNA-packaged liposomes and expression of liposome-delivered plasmid DNA in target cells is that metaphyseal cells with highly metabolic activity ${ }^{1}$ are more prone to uptake and express plasmids DNA than cells in the diaphysis of tibia and non-skeletal tissues. Consistent with this observation, no detectable histological alterations in non-skeletal organs such as the heart, liver, spleen, lung, or kidneys are observed after a 6week course of systematic treatment of OVX mice with DNApackaged cationic liposomes, supporting the absence of liposomeZEB1-GFP- or liposome-Vector-GFP-induced toxicity or side effects. ZEB1 is inappropriately upregulated in a variety of malignant tumor types and functions as a stimulating factor of tumor invasion and metastasis ${ }^{10-13}$. Whether ectopic expression of ZEB1 in normal cells (e.g., metaphyseal cells) could induce transformation of the cells is still elusive. Future work will determine whether the extended period of treatment with Zeb1packaged liposomes is still safe to the animals.

In conclusion, our results more broadly suggest that Zeb1packaged cationic liposomes targeting bone vasculature may have a synergistic or complementary effect when used in combination with an osteoblast-targeted anabolic such as a parathormone analog or an anti-sclerostin antibody. The findings presented here lay the foundation for new therapeutic strategies in the treatment of osteoporotic disease states by promoting angiogenesisdependent osteogenesis.

\section{Methods}

Mice. Mice were housed under standard specific-pathogen-free conditions and all animal experiments were performed in accordance with protocols that were approved and authorized by the Animal Welfare and Ethics Committee of China Pharmaceutical University. Mice carrying Zeb1 (exon 3) floxed alleles (Zeb $1^{f l f l}$ ) were generated in our laboratory and have been described previously ${ }^{15}$. Tie2-Cre transgenic mice were purchased from Jackson Laboratory (\#008863). Cdh5(PAC) CreERT2 transgenic mice were kindly provided by Ralf H. Adams (Max Planck 
a
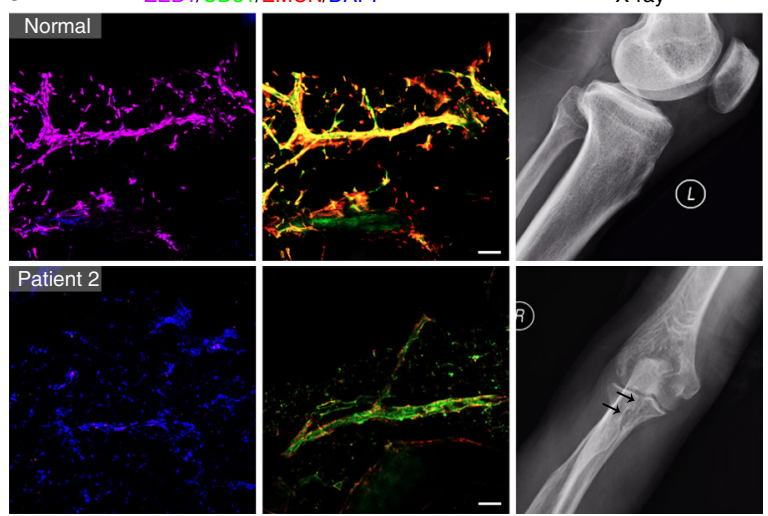

(1)

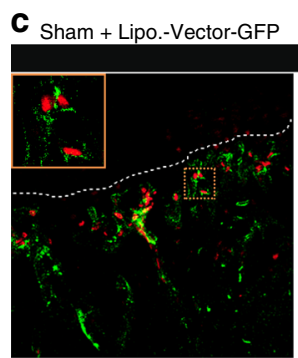

OVX + Lipo.-Vector-GFP

OVX + Lipo.-ZEB1-GFP
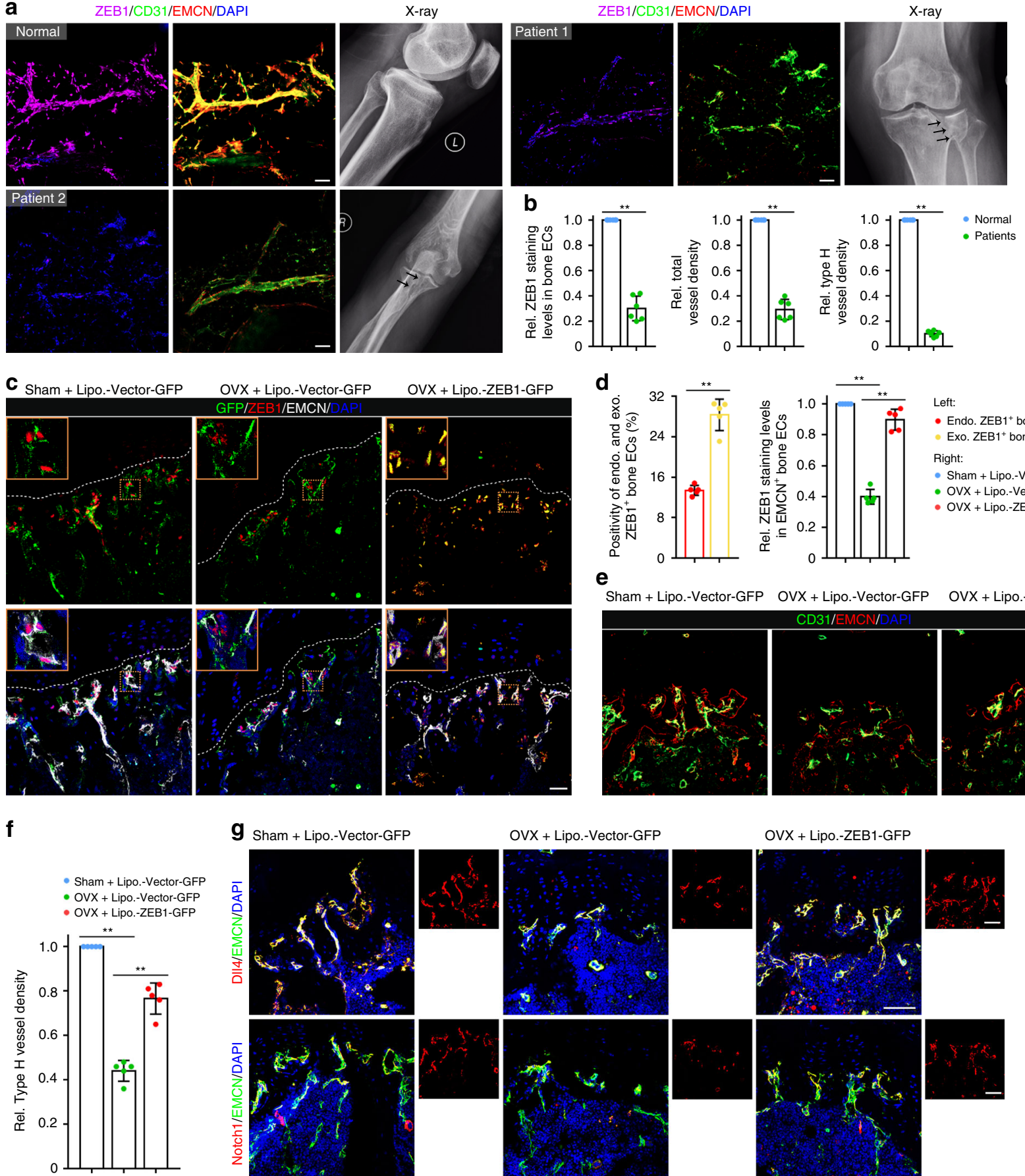

Left:

- Endo. ZEB1+ bone ECs

Exo. ZEB1 ${ }^{+}$bone ECs

Right:

- Sham + Lipo-Vector-GFP

OVX + Lipo.-Vector-GFP

OVX + Lipo.-ZEB1-GFP

e

Sham + Lipo.-Vector-GFP OVX + Lipo.-Vector-GFP OVX + Lipo.-ZEB1-GFP

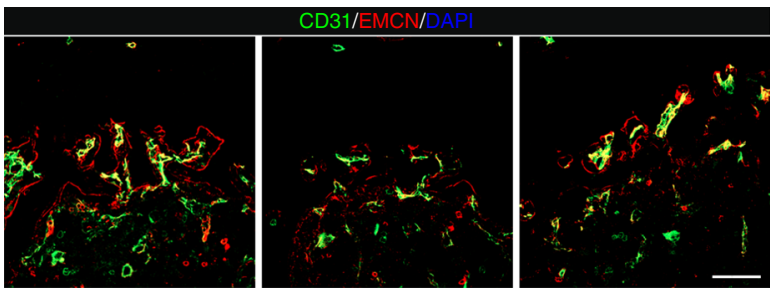

g Sham + Lipo.-Vector-GFP

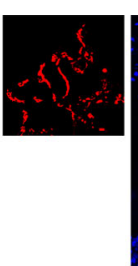

OVX + Lipo.-Vector-GFP
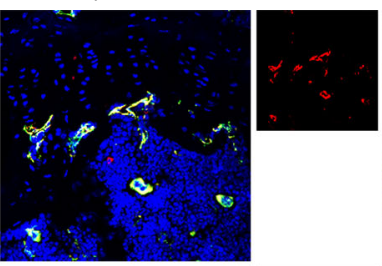

OVX + Lipo.-ZEB1-GFP
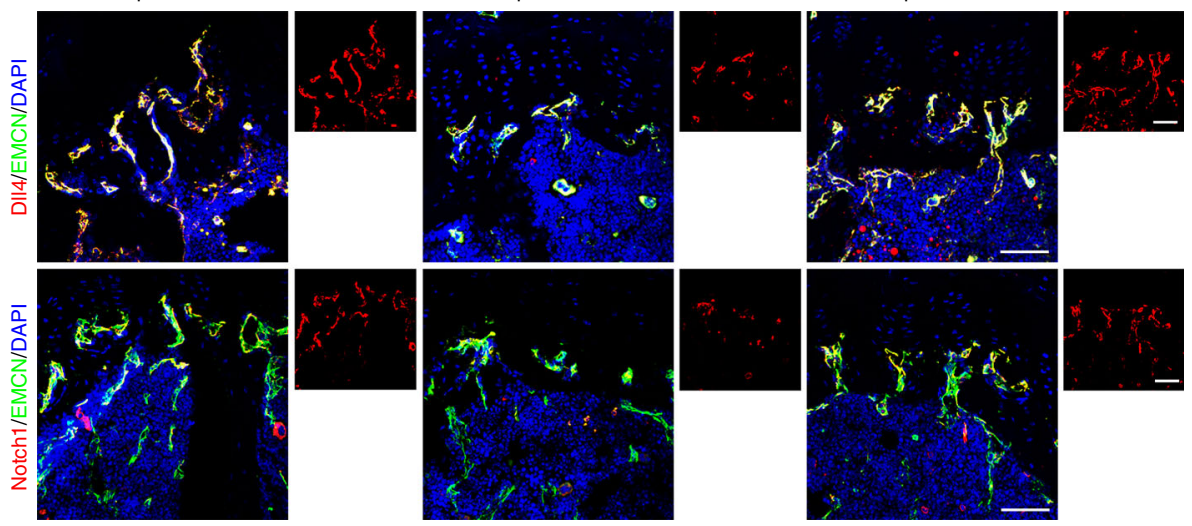

f

h

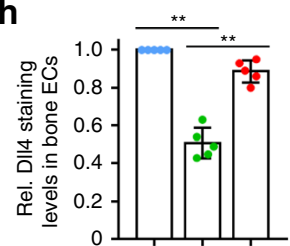

Institute for Molecular Biomedicine, Münster, Germany ${ }^{18}$ ). For generation of intrinsic and inducible EC-specific ZEB1-deleted mice, Zeb $1^{f l / f l}$ mice were bred with Tie2-Cre and Cdh5(PAC)-CreERT2 transgenic mice, respectively. To induce Cdh5(PAC)-CreERT2 activity and gene inactivation in pups, mice at P8 were i.p. injected with $0.1 \mathrm{mg}$ tamoxifen $(10 \mu \mathrm{l}, 10 \mathrm{mg} / \mathrm{ml}$, dissolved in 1:10 ethanol/corn oil; Sigma-Aldrich, \#T5648) every day for 7 consecutive days. To induce gene inactivation in adults, 7 -week-old mice were i.p. injected with $1.0 \mathrm{mg}$ tamoxifen $(100 \mu \mathrm{l}$, $10 \mathrm{mg} / \mathrm{ml}$ ) every other day for 2 consecutive weeks. For r.Dll4 administration experiments, $Z e b 1^{W T}$ and $Z e b 1^{\triangle E C}$ mice were i.p. injected with $1 \mu \mathrm{g} / \mathrm{g}$ r.Dll4 protein (R\&D Systems, \#1389-D4-050) at P4 for 2 consecutive weeks before analysis at P21. To generate OVX and Sham mouse models, the surgery was performed on 8 week-old female mice, and the mice at day 3 post surgery were i.v. injected with $4 \mu \mathrm{g}$ Lipo.-Vector-GFP or Lipo.-ZEB1-GFP twice every week for consecutive 6 weeks. Mice were then killed and uterine, long bone, and non-skeletal organs were dissected for further use. All mice were kept in C57BL/6J background and gender-matched littermate controls were used in all experiments. The investigators 
Fig. 7 Administration of OVX mice with Zeb1-packaged cationic liposomes efficiently restores the impaired bone angiogenesis. a Representative confocal images of ZEB1/CD31/EMCN immunostaining in the tibia of normal and osteoporotic patients ( $n=6$, each). Arrow in X-ray image depicts tibial osteoporotic fracture undergoing knee joint replacement. Scale bar, $30 \mu \mathrm{m}$. b Quantification of ZEB1 staining levels in bone ECs, total vessel density, and type $\mathrm{H}$ vessel density in the tibia as shown in a. c Representative confocal images of GFP/ZEB1/EMCN immunostaining in the metaphysis of the tibia of Sham and OVX mice that were i.v. injected with $4 \mu$ g Lipo.-Vector-GFP or Lipo.-ZEB1-GFP for 6 consecutive weeks (designed Sham + Lipo.-Vector-GFP, OVX + Lipo.-Vector-GFP, and OVX + ZEB1-GFP mice, respectively; $n=5$, each). Scale bar, $100 \mu \mathrm{m}$. $\mathbf{d}$ Quantification of positivity of endogenous and exogenous ZEB1+ bone ECs (left panel) and ZEB1 staining levels in $\mathrm{EMCN}^{+}$bone ECs (right panel) in the tibia as shown in $\mathbf{c}(n=5$ independent experiments). e Representative confocal images of CD31/EMCN immunostaining in the tibia as shown in $\mathbf{c}(n=5$, each). Scale bar, $60 \mu \mathrm{m}$. $\mathbf{f}$ Quantification of type $\mathrm{H}$ vessel density in the tibia as shown in $\mathbf{c}(n=5$ independent experiments). $\mathbf{g}$ Representative confocal images of DII4/EMCN (top panels) and Notch1/EMCN (bottom panels) immunostaining in tibia as shown in $\mathbf{c}(n=5$, each). Scale bar, $60 \mu \mathrm{m}$. h Quantification of DII4 (left panel) and Notch1 (right panel) staining levels in bone ECs of the tibia as shown in $\mathbf{g}\left(n=5\right.$ independent experiments). All data are represented as mean \pm SD. ${ }^{\star \star} P<0.01$. Differences are tested using unpaired two-tailed Student's $t$-test (b) and one-way ANOVA with Tukey's post hoc test (d, $\mathbf{f}, \mathbf{h})$. The source data are provided as a Source Data file.

a Sham + Lipo.-Vec.-GFP
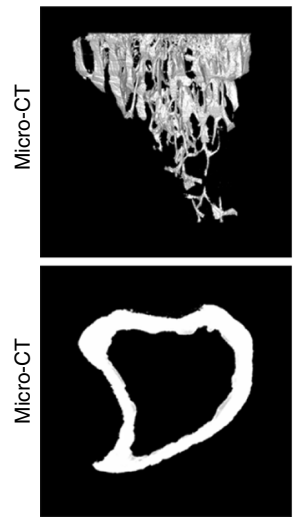

OVX + Lipo.-Vec.-GFP
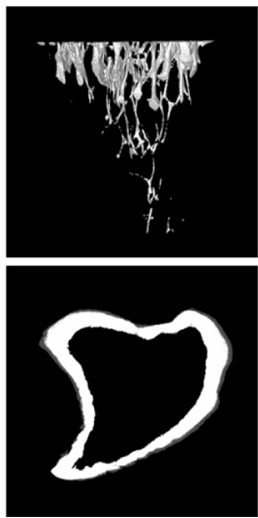

OVX + Lipo.-ZEB1-GFP
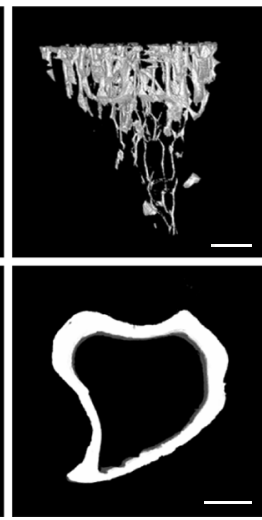

b

Sham + Lipo.-Vec.-GFP

- OVX + Lipo.-Vec.-GFP.
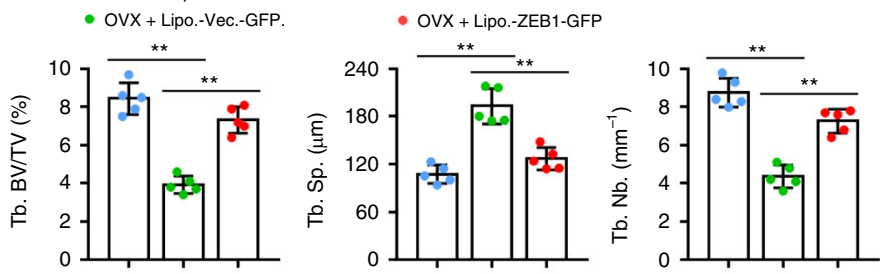

C
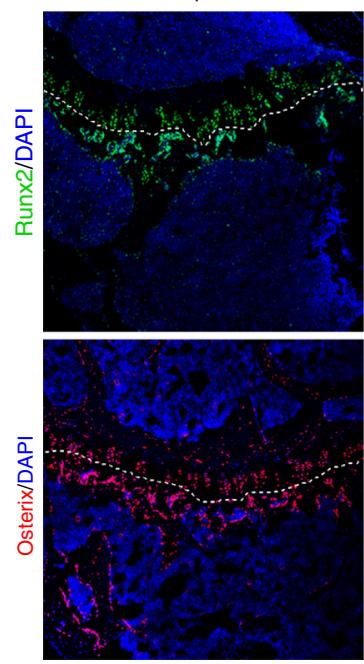

OVX + Lipo.-Vec.-GFP
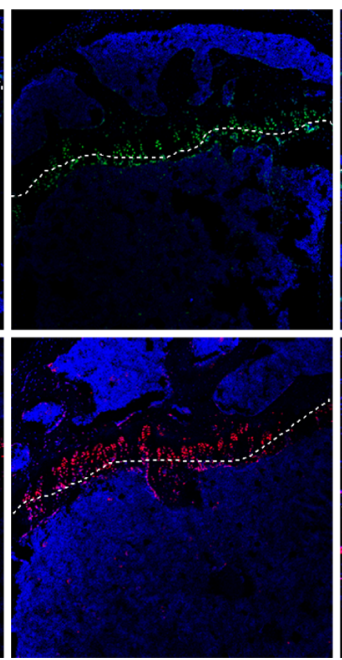

OVX + Lipo.-ZEB1-GFP
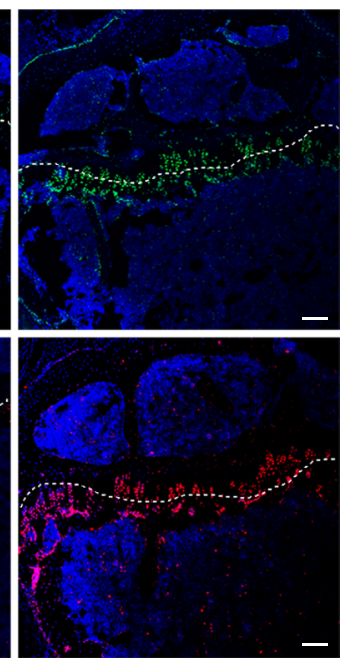

d
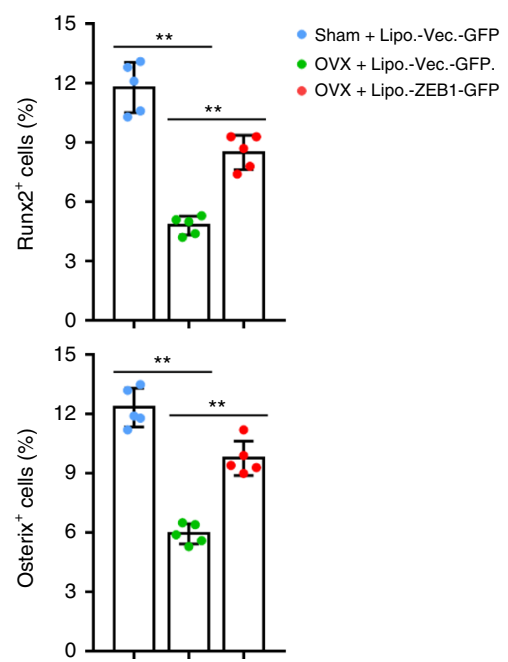

Fig. 8 Treatment with Zeb1-packaged cationic liposomes has therapeutic effects on OVX-induced bone loss. a Representative micro-CT images of trabecular bone (top panels) and cortical bone (bottom panels) of the tibia in Sham and OVX mice that were i.v. injected with $4 \mu \mathrm{g}$ Lipo.-Vector-GFP or Lipo.-ZEB1-GFP for 6 consecutive weeks (designed Sham + Lipo.-Vector-GFP, OVX + Lipo.-Vector-GFP, and OVX + ZEB1-GFP mice, respectively; $n=5$, each). Scale bar, $0.2 \mathrm{~mm}$. b Quantification of bone architectures in the tibia as shown in $\mathbf{a}(n=5$ independent experiments). c Runx 2 and Osterix immunostaining of the tibia as described in $\mathbf{a}\left(n=5\right.$, each). Scale bar, $100 \mu \mathrm{m}$. d Quantification of Runx $2^{+}$and Osterix ${ }^{+}$cells in the tibia as shown in c $\left(n=5\right.$ independent experiments). All data are represented as mean \pm SD. ${ }^{\star \star} P<0.01,{ }^{\star} P<0.05$. Differences are tested using one-way ANOVA with Tukey's post hoc test $(\mathbf{b}, \mathbf{d})$. The source data are provided as a Source Data file.

were not blinded to allocation during experiments and outcome assessment. No method of randomization was used as mice were segregated into groups based on genotype alone. No statistical method was used to predetermine sample size.

Genotyping. Tail biopsy $(\sim 2 \mathrm{~mm})$ samples were digested at $95^{\circ} \mathrm{C}$ for $30 \mathrm{~min}$ in $50 \mu \mathrm{l}$ of buffer $1(10 \mathrm{~N} \mathrm{NaOH}$ and $0.5 \mathrm{M}$ EDTA pH 12.0). An equal amount of buffer 2 (40 mM Tris-HCl pH 5.0) was added to neutralize buffer 1 . The mixtures were immediately vortexed and centrifuged at $12,000 \times g$ for $5 \mathrm{~min}$. The supernatants (containing genomic DNAs) were collected and stored in $-20^{\circ} \mathrm{C}$ for further use. One microliter of extracted genomic DNA was added to $20 \mu \mathrm{l}$ of PCR reaction mixture containing $10 \mu \mathrm{l} 2 \times$ GoTaq Green Master Mix (Promega, \#M7123), $0.5 \mu \mathrm{l}$ forward/reverse primers, and $8.5 \mu \mathrm{l}$ RNase/DNase free $\mathrm{H}_{2} \mathrm{O}$. Primer sequences for amplifying the indicated mouse transgenes are as follows. 
Zeb1-flox/wt (flox-296 bp, wt-197 bp),

5'-GGTTTTACCATGCCCACTAATATGA-3'/5'-GAGGCAAGAAAAACAA

ATGTAATCTCC-3';

Tie2-Cre (PCR product, $450 \mathrm{bp}$ ),

5'-AGGTGTAGAGAAGGCACTTAGC-3'/5'-CTAATCGCCATCTTCCAGGA

GG-3';

Cdh5(PAC)-CreERT2 (PCR product, $720 \mathrm{bp}$ ),

5'-GCCTGCATTACCGGTCGATGCAACGA-3'/5'-GTGGCAGATGGCGCG GCAACACCATT-3'.

Immunofluorescence and histology. For immunofluorescence, tissues were freshly dissected and fixed in $4 \%$ paraformaldehyde (PFA)-phosphate-buffered saline (PBS) at $4{ }^{\circ} \mathrm{C}$ for $4 \mathrm{~h}$ with constant shaking. Bone samples were decalcified in $0.5 \mathrm{M}$ EDTA at $4{ }^{\circ} \mathrm{C}$ and the decalcified bones were immersed into $20 \%$ sucrose and $2 \%$ polyvinylpyrrolidone solution for $24 \mathrm{~h}$. Bone and non-skeletal tissues were embedded in OCT (Sakura, \#4583) and $50 \mu \mathrm{m}$-thick sections were prepared. Paraffin-embedded sections were deparaffinized and treated with the Antigen Unmasking Solution (\#H-3300, Vector). Tissue sections were permeabilized for $15 \mathrm{~min}$ in $0.3 \%$ Triton X-100. After washing, sections were blocked with $5 \%$ donkey serum (in PBS) at room temperature for $30 \mathrm{~min}$ and incubated overnight at $4{ }^{\circ} \mathrm{C}$ with primary antibodies. Cryostat sections were air-dried, permeabilized in $0.3 \%$ Triton X-100 for $15 \mathrm{~min}$, blocked in 5\% goat or donkey serum at room temperature for $30 \mathrm{~min}$, and probed with primary antibodies diluted in $5 \%$ goat or donkey serum in PBS overnight at $4{ }^{\circ} \mathrm{C}$. The following antibodies were used: ZEB1 (Santa Cruz, \#sc-25388, 1:200), Endomucin (Santa Cruz, \#sc-65495, 1:200), CD31 conjugated to Alexa Fluor 488 (R\&D Systems, \#FAB3628G, 1:100), CD31 (BD Pharmingen, \#553370, 1:200), Osterix (Santa Cruz, \#sc-22536, 1:200), Runx2 (Abcam, \#ab192256, 1:200), VEGFA (Abcam, \#ab52917, 1:200), Notch1 (Cell Signaling, \#4380, 1:100), Dll4 (R\&D Systems, \#AF1389, 1:100), NICD1 (Abcam, \#ab8925, 1:100), ALP (R\&D Systems, \#MAB29091, 1:200), and GFP (Abcam, \#ab5450, $1: 200)$. After incubation with primary antibodies, sections were washed with PBS three times and were incubated with species-appropriate Alexa Fluor-coupled secondary antibodies (all from Abcam, 1:200) for $1 \mathrm{~h}$ at room temperature. Sections were then counterstained with 4',6-diamidino-2-phenylindole (DAPI) and images were acquired on a Zeiss LSM800 microscope.

For calcein double labeling, mice were i.p. injected with $10 \mathrm{mg} / \mathrm{kg}$ calcein (Sigma-Aldrich, \#C0875) dissolved in $2 \%$ sodium bicarbonate solution at P13 and $\mathrm{P} 19$, and bone samples were collected at P21, fixed in 4\% PFA, directly embedded in methyl methacrylate, and sagittally sectioned at $30 \mu \mathrm{m}$. Images of calcein double labeling in undecalcified tibial sections were acquired on a Zeiss LSM800 confocal microscope.

For histology, non-skeletal tissues were fixed in 4\% PFA-PBS and embedded in paraffin. Bone samples were fixed in $4 \%$ PFA-PBS, decalcified as described above, and embedded in paraffin. The embedded tissues were sectioned at $5 \mu \mathrm{m}$, deparaffinized, and subjected to hematoxylin and eosin staining and tartrateresistant acid phosphatase (TRAP) staining using an acid phosphatase leukocyte kit (Sigma-Aldrich, \#386 A) according to the manufacturer's instruction. In some experiments, bone samples were fixed in $4 \%$ PFA-PBS, directly embedded in paraffin, sectioned, and subjected to Alizarin red S staining (Sigma-Aldrich, \#A5533).

Micro-CT analysis. The tibias were freshly dissected, fixed in 4\% PFA-PBS, and analyzed using high-resolution micro-CT (Hiscan Information Technology, \#Hiscan-H100) and software Hiscan Analyzer V3.0. The scanner was set at a voltage of $70 \mathrm{kV}$, a current of $110 \mathrm{~mA}$, and a resolution of $10.8 \mu \mathrm{m} /$ pixel. The metaphysis of the tibia was scanned and metaphyseal parameters such as $\mathrm{Tb}$. BV/TV, Tb. Th., trabecular separation (Tb. Sp.), Tb. Nb., and cortical bone thickness (Ct. Th.) were measured.

Isolation and culture of bone ECs. To isolate primary bone ECs, the tibias were collected in sterile $\mathrm{Ca}^{2+}$ and $\mathrm{Mg}^{2+}$ free $\mathrm{PBS}$, crushed, and digested in collagenase A (Sigma-Aldrich, \#SCR136) for $30 \mathrm{~min}$ at $37^{\circ} \mathrm{C}$ to obtain a single-cell suspension. Bone ECs were then magnetic activated cell sorting sorted using Endomucin antibody (Santa Cruz, \#sc-65495) and Dynabeads sheep anti-Rat IgG (Thermo Fisher, \#11035). Sorted bone ECs were seeded on fibronectin-precoated culture dishes and cultured in EBM-2 EC growth medium (Lonza, \#00190860) containing supplements and growth factors (Lonza, \#CC-4176). Normally, bone ECs were used within five passages. In some experiments, bone ECs isolated from the tibia of $\mathrm{ZEB1}^{\mathrm{fl} / \mathrm{fl}}$ mice were infected with recombinant adenovirus expressing $\beta \mathrm{Gal}$ (Vigene Biosciences, \#CV1001) or Cre (\#CV10010) at a multiplicity of infection of 200 for $24 \mathrm{~h}$ and re-infected with for additional $24 \mathrm{~h}$ to generate control or ZEB1-deleted cells.

Flow cytometry. Tibias were collected in sterile $\mathrm{Ca}^{2+}$ and $\mathrm{Mg}^{2+}$ free PBS, crushed, and digested in collagenase A (Sigma-Aldrich, \#SCR136) for $30 \mathrm{~min}$ at $37^{\circ} \mathrm{C}$ to obtain a single-cell suspension. Equal number of cells were immunostained with Endomucin antibody (Santa Cruz, \#sc-65495) for $45 \mathrm{~min}$ at $4^{\circ} \mathrm{C}$. After washing, cells were immunostained with PE-CD45 (BD Pharmingen, \#553081), APC-Ter119 (BD Pharmingen, \#557909), PE-Cy7-CD31 (BD Pharmingen, \#561410), and Alexa Fluor 647 goat anti-rat secondary antibody (Thermo Fisher, \#A-21247) for $45 \mathrm{~min}$ at $4{ }^{\circ} \mathrm{C}$, followed by DAPI staining for $5 \mathrm{~min}$ before analysis and sorting. Finally, cells were acquired on a BD FACS Verse flow cytometer and sorted on a FACS Aria II flow cytometer. To demarcate and sort CD $31^{\text {hi }}{ }^{2 M C N}{ }^{\text {hi }}$ cells, first standard quadrant gates were set. To differentiate CD31 ${ }^{\text {hi }} \mathrm{EMCN}^{\mathrm{hi}}$ cells from the total double positive cells in quadrant, two gates were set at $>10^{4} \log$ Fl-2 (PE-Cy7-CD31) fluorescence and $>10^{4}$ log Fl-4 (Alexa Fluor 647-Endomucin) fluorescence. DAPI ${ }^{-} \mathrm{CD} 45^{-}$Ter $119^{-} \mathrm{CD} 31^{+}$cells were sorted according to side scatter and were set at $>10^{3} \log$ Fl-2 fluorescence (PE-Cy7-CD31) after negative selection of CD45 and Ter119 at $<10^{3} \log$ Fl-2 (PE-CD45 and APC-Ter119) fluorescence.

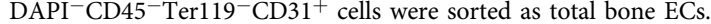

Quantitative reverse-transcriptase PCR. Total RNA was isolated and reversely transcribed using the RNeasy kit (Qiagen, \#74104) and the PrimeScript RT reagent kit (TaKaRa, \#RR037A), respectively, according to the manufacturer's instructions qPCR was performed using the SYBR Green PCR Master Mix (Vazyme, \#Q341) and relative mRNA expression was normalized to Gapdh. Primer sequences are as follows.

Zebl, $5^{\prime}$-TTATCCTGAGGCGCCCGAGGA-3'/5'-TACGGGCAGGTGAGCAA CTGG-3';

Zeb2, 5'-GCACCCAGCTCGAGAGGCAT-3' $/ 5^{\prime}$-AAGGCCTTGCCACACTCC GTG-3';

Notch $1,5^{\prime}$-TGCCTGAATGGAGGTAGGTGC-3' $/ 5^{\prime}$-GCACAGCGATAGGAG CCGATC-3';

Notch2, 5' -TGACTGTTCCCTCACTATGG-3'/5'-CACGTCTTGCTATTCCTC T-3';

Notch3, 5' -TGCCAGAGTTCAGTGGTGG-3'/5' -TGCCAGAGTTCAGTGGT

GG-3';

Notch4, 5' -CTCTTGCCACTCAATTTCCCT-3'/5' -TTGCAGAGTTGGGTATC CCTG-3';

Dll4, 5'-GACTGAGCTACTCTTACCGGG-3's' $5^{\prime}$-CTTACAGCTGCCACCATT TCG-3';

Hes $1,5^{\prime}$-GGAGAAGAGGCGAAGGGCAAG-3'/5'-GGTTCCGGAGGTGCTT CACAG-3';

Hes $5,5^{\prime}$-GAAACACAGCAAAGCCTTCG-3' $/ 5^{\prime}$-AGCTTCATCTGCGTGTC G-3';

Hey1, 5'-GGCTGGTACCCAGTGCCTTTG-3'/5'-CCTTTCCCTCCTGCAGT

GTGC-3';

Heyl, 5'-CAGCCCTTCGCAGATGCAA-3'/5'-CCAATCGTCGCAATTCAGA AAG- $3^{\prime}$;

Efnb2, 5' -TGGGTCTTTGGAGGGCCTGGAT-3'/5'-GGACCGTGATTCCTG GCTGATC-3';

$T g f b 1,5^{\prime}$-CTCCCGTGGCTTCTAGTGC-3' $15^{\prime}$-GCCTTAGTTTGGACAGGAT CTG-3';

Tgfb2, $5^{\prime}$-TCGACATGGATCAGTTTATGCG-3' ${ }^{\prime} / 5^{\prime}$-CCCTGGTACTGTTGTA GATGGA-3';

Tgfb3, 5' -CAGGCCAGGGTAGTCAGAG-3's' ${ }^{\prime}$-ATTTCCAGCCTAGATCCTG CC-3';

Bmp2, 5'-GGGACCCGCTGTCTTCTAGT-3'/5'-TCAACTCAAATTCGCTGA GGAC-3';

Bmp4, 5'-TTCCTGGTAACCGAATGCTGA-3' $/ 5^{\prime}$-CCTGAATCTCGGCGACT

TTTT-3';

$P g f, 5^{\prime}$-TCTGCTGGGAACAACTCAACA-3'/ $5^{\prime}$-GTGAGACACCTCATCAGG GTAT- ${ }^{\prime}$; G-3';

Fgf $1,5^{\prime}$-CCCTGACCGAGAGGTTCAAC-3'/5'-GTCCCTTGTCCCATCCAC Vegfa, 5' -GCACATAGAGAGAATGAGCTTCC-3' $/ 5^{\prime}$-CTCCGCTCTGAACAA GGCT-3';

Nog, 5'-GCCAGCACTATCTACACATCC-3'/5'-GCGTCTCGTTCAGATCCT TCTC-3';

Gapdh, 5' -CCCTGGCCAAGGTCATCCATG-3'/5' -TGATGTTCTGGGCAGC CCCAC-3'

Immunoblot and immunoprecipitation assays. Immunoblot assay was performed as described previously ${ }^{15}$. Briefly, cells were collected and lysed in RIPA buffer (Thermo Fisher, \#89900) containing protease inhibitor cocktail (Thermo Fisher, \#87786). In some experiments, nuclear proteins were extracted using the NE-PER Nuclear and Cytoplasmic Extraction Reagents (Thermo Fisher, \#78833) according to the manufacturer's instructions. The cell lysates were subjected to immunoblot assay using primary antibodies against ZEB1 (Santa Cruz, \#sc-25388, 1:1,000), Dll4 (R\&D Systems, \#AF1389, 1:1,000), Notch1(Cell Signaling, \#4380, 1:1,000), NICD1 (Cell Signaling, \#4147, 1:500), CBP (Cell Signaling, \#7389, 1:1,000), p300 (Cell Signaling, \#70088, 1:1,000), HA (Cell Signaling, \#3724, 1:1,000), HDAC1 (Cell Signaling, \#2062, 1:1,000), and $\beta$-actin (Santa Cruz, \#sc$47778,1: 2,000)$. IP assay was performed as described previously ${ }^{15}$. In brief, cells were collected and lysed in IP lysis buffer $(50 \mathrm{mM}$ Tris- $\mathrm{HCl}, 150 \mathrm{mM} \mathrm{NaCl}, 1 \mathrm{mM}$ EDTA, 1\% NP40, pH 7.4) supplemented with protease inhibitor cocktail for $20 \mathrm{~min}$ on ice. The cell lysates were sonicated, clarified, and incubated with anti-ZEB1 antibody (Santa Cruz, \#sc-25388, 1:200) followed by incubation with pre-cleared Protein A/G agarose beads (Santa Cruz, \#sc-2003). The immunocomplexes were 
subjected to immunoblotting using antibodies against CBP, p300, and ZEB1. The original uncropped scans of western blottings are shown in Source Data file.

ChIP and sequential ChIP assays. Bone ECs were fixed in $1 \%$ formaldehyde for $10 \mathrm{~min}$, quenched in $0.125 \mathrm{M}$ glycine for $5 \mathrm{~min}$ at room temperature, washed with ice-cold PBS for three times, and lysed in lysis buffer $(50 \mathrm{mM}$ Tris- $\mathrm{HCl}, 150 \mathrm{mM}$ $\mathrm{NaCl}, 1 \mathrm{mM}$ EDTA, $1 \% \mathrm{NP} 40 \mathrm{pH}$ 7.4) supplemented with protease inhibitor cocktail for $20 \mathrm{~min}$ on ice. The cell lysates were sonicated until the sheared DNA was $\sim 200-1000$ bp in size. After centrifugation, the supernatants were pre-cleared with protein $\mathrm{A} / \mathrm{G}$ beads and saturated with salmon sperm DNA (Thermo Fisher, $\# 15632011)$ at $4^{\circ} \mathrm{C}$ for $1 \mathrm{~h}$. Five percent of the sheared chromatin was used as input control and the rest was incubated overnight with the antibodies against ZEB (Santa Cruz, \#sc-25388), acetyl-histone H3 (K4; Cell Signaling, \#7627), acetylhistone H3 (K18; Cell Signaling, \#9675), acetyl-histone H3 (K14; Abcam, \#ab176799), and tri-methyl-histone $\mathrm{H} 3$ (K27; Cell Signaling, \#9377) at $4{ }^{\circ} \mathrm{C}$ under rotation, followed by another incubation with protein A/G beads (Santa Cruz, \#sc2003) for $1 \mathrm{~h}$ at $4{ }^{\circ} \mathrm{C}$. Beads-bound chromatins were sequentially washed with lowsalt wash buffer once, high-salt wash buffer once, and TE buffer twice, and were eluted with elution buffer ( $1 \% \mathrm{SDS}, 0.1 \mathrm{M} \mathrm{NaHCO}_{3}$ ) twice. The eluted DNAprotein complexes were incubated with $0.2 \mathrm{M} \mathrm{NaCl}$ overnight at $65^{\circ} \mathrm{C}$, RNase A for $30 \mathrm{~min}$ at $37^{\circ} \mathrm{C}$, and proteinase $\mathrm{K}$ (Thermo Fisher, \#AM2548) for $1.5 \mathrm{~h}$ at $45^{\circ} \mathrm{C}$. The bound DNA was purified using a kit (Sigma-Aldrich, \#NA1020) according to the manufacturer's instructions and then subjected to PCR or qPCR analysis. For sequential ChIP assay, following sequential washes, half amount of beads were eluted as described above and saved for further use. Another half amount of beads were eluted with $2 \%$ dithiothreitol buffer at $37^{\circ} \mathrm{C}$ for $30 \mathrm{~min}$. The supernatants were collected and incubated with anti-CBP antibody (Cell Signaling, \#7389) or IgG isotope control (Santa Cruz, \#sc-2763) overnight at $4{ }^{\circ} \mathrm{C}$, followed by another incubation with protein $\mathrm{A} / \mathrm{G}$ beads for $1 \mathrm{~h}$ at $4{ }^{\circ} \mathrm{C}$. Beads-bound chromatins were sequentially washed with low-salt wash buffer once, high-salt wash buffer once and TE buffer twice and eluted with elution buffer (1\% SDS, $\left.0.1 \mathrm{M} \mathrm{NaHCO}_{3}\right)$ twice. The eluted DNA-protein complexes and the resulting bound DNA were treated as described above. Primer sequences are as follows.

Dll4-proximal (PCR product, $81 \mathrm{bp}$ ),

5'-CAGCCTCAAGCTCTCTCACC-3'/5'-CGCTGGAGTAGGGAGGAAAC T-3'

Dll4-distal (PCR product, $90 \mathrm{bp}$ )

5'-TTGCCTAGAGGGAAAGAAAGG-3'/5'-GCGATCTGGGAGACTGTATT G-3'.

Notch1-proximal (PCR product, $104 \mathrm{bp}$ ),

5'-GCCAGGGCGCCACATTTAAAC-3'/5'-GAACCAGCTCCATCCTGGA GT-3'

Notch1-distal (PCR product, $85 \mathrm{bp}$ ),

5'-AAGGGGAGCGAGTGAATGAGG-3'/5'-GCTCAGTACCAGCTCAGCC TT-3'.

Luciferase reporter assay. The $\sim 1.1 \mathrm{~kb}$ fragment of WT or MUT mouse Dll4 promoter $(-1018 \sim+76)$ and the $\sim 1.2 \mathrm{~kb}$ fragment of WT or MUT mouse Notch 1 promoter $(-1039 \sim+182)$ were synthesized by GenScript and subcloned into the pGL3 basic luciferase reporter vector (Promega, \#E1751). Control or ZEB1-deleted bone ECs were eletroporated with Dll4-WT (or Dll4-MUT, Notch1-WT, or Notch1MUT) reporter constructs and pRL-TK Renilla luciferase control construct. Bone ECs were also eletroporated with pcDNA3.1 (or pcDNA3.1-HA-ZEB1), Dll4-WT (or Dll4-MUT, Notch1-WT, or Notch1-MUT) reporter constructs, and pRL-TK Renilla luciferase control construct. HEK293T cells were transfected with pcDNA3.1, pcDNA3.1-HA-ZEB1, pSG5-HA-p300 (Addgene, \#89094), pRC/RSVmCBP-HA (Addgene, \#16701), ZEB1 + p300, ZEB1 + CBP together with Dll4-WT or Notch1-WT promoter reporter constructs, and pRL-TK Renilla luciferase control construct. Dual-luciferase reporter assays were performed according to the manufacturer's instructions (Promega, \#E1910).

r.Dll4 treatment. Culture dishes were precoated with $500 \mathrm{ng} / \mathrm{ml}$ r.Dll4 protein (R\&D Systems, \#1389) dissolved in PBS overnight at $4{ }^{\circ} \mathrm{C}$ and control and ZEB1deleted bone ECs were seeded on r.Dll4-precoated culture dishes and grown for $24 \mathrm{~h}$. Cells were collected and total RNAs were isolated for RT-qPCR analysis.

Human bone samples. Human bone samples were obtained from patients (female) based on the inclusion and exclusion criteria. Six patients with osteoporosis undergoing knee joint replacement with ages ranging from 58 to 75 years and six patients with tibia fracture undergoing Open Reduction Internal Fixation ranging in age from 12 to 30 years (human bone samples were collected at the Division of Orthopedic Surgery, the Affiliated Nanjing Hospital, Nanjing Medical University, Nanjing, China). This clinical study was approved by the Ethnic Committee of the Affiliated Nanjing Hospital, Nanjing Medical University, and written informed consents were obtained from the patients before procedure.

DNA-packaged liposome preparation and characterization. Full length of murine Zeb1 cDNA were synthesized by GenScript and subcloned into pcDNA3.1 $+\mathrm{C}$-eGFP (designed Vector-GFP) vector to generate pcDNA3.1+C-eGFP-ZEB1 (designed ZEB1-GFP). For preparation of cationic liposomes, 1,2-dioleoyl-sn-glycero-3-phosphoethanolamine, 1,2-dioleoyl-3-trimethylammonium propane, and cholesterol were dissolved in chloroform at a molar ratio of 4:3:3, evaporated in a rotary evaporator at $40^{\circ} \mathrm{C}$ for $30 \mathrm{~min}$, dried overnight, hydrated with dd $\mathrm{H}_{2} \mathrm{O}$ at $40^{\circ} \mathrm{C}$ for $20 \mathrm{~min}$, and finally ultrasounded at an intensity of $15 \%$. Vector-GFP $(0.2$ $\mathrm{mg} / \mathrm{ml})$ or ZEB1-GFP $(0.2 \mathrm{mg} / \mathrm{ml})$ was mixed with cationic protamine at different $\mathrm{N}: \mathrm{P}$ ratios (the molar ratio of nitrogen in protamine to phosphate in DNA vector) to form the nano-sized DNA/protamine complex (designed Protamine-VectorGFP and Protamine-ZEB1-GFP). The Protamine-Vector-GFP and ProtamineZEB1-GFP was subsequently mixed with cationic liposomes at $60 \mathrm{nmol}$ lipid/ $\mathrm{\mu g}$ DNA to generate the final Lipo.-Vector-GFP and Lipo.-ZEB1-GFP, respectively. DNA packing efficiency was examined by agarose gel electrophoresis. Particle size and zeta potential were measured using a Zetasizer NANO ZSP (Malvern Panalytical). For preparation of fluorescence-loaded liposomes, $\mathrm{N}$-(lissamine rhodamine B sulfonyl)-1,2-dihexadecanoyl-sn-glycero-3-phosphoethanolamine (2 mol\%) and $\operatorname{DiR}(0.1 \mathrm{~mol} \%)$ were added in the lipid components.

Pharmacokinetics. For pharmacokinetic analysis, pcDNA3.1+C-eGFP control vector or pcDNA3.1+C-eGFP-ZEB1 vector was packaged into rhodamine PElabeled liposome and the DNA-packaged liposomes (designed Rho.-Lipo.-VectorGFP and Rho.-Lipo.-ZEB1-GFP, respectively) were i.v. injected into

Sprague-Dawley rats $(n=5$, each) at a dose of $0.8 \mathrm{mg} / \mathrm{kg}$; The blood samples collected at $0,0.125,0.25,0.5,1,2,4,8,12$, and $24 \mathrm{~h}$ post treatment, were centrifuged, and the supernatant plasma was collected for fluorescence intensity measurement at the excitation wavelength of $552 \mathrm{~nm}$ and the emission wavelength of $610 \mathrm{~nm}$ on a SpectraMax iD5 Multi-Mode Microplate Reader (Molecular Devices). The fluorescence intensity-time curves of Rho.-Lipo.-Vector-GFP and Rho.-Lipo.-ZEB1-GFP were plotted. The pharmacokinetic parameters such as elimination half-life $\left(t_{1 / 2}, \mathrm{~h}\right)$, maximal plasma concentration $\left(C_{\max }, \times 10^{4}\right.$ a.u. $\left./ \mathrm{ml}\right)$, area under the plasma concentration-time curve $\left(\mathrm{AUC}_{0-\infty} \times 10^{4}\right.$ a.u. $\left./ \mathrm{ml} \times \mathrm{h}\right)$, and MRT (h) were calculated using Phoenix WinNonlin 6.4 software.

Biodistribution measurement. For biodistribution analysis, pcDNA3.1 + CeGFP-ZEB1 vector was packaged into DiR-labelled liposome and the DNApackaged liposomes (designed DiR-Lipo.-ZEB1-GFP) were i.v. injected into 8-week-old C57BL6 mice at a dose of $1.0 \mathrm{mg} / \mathrm{kg}$; the kidneys, spleen, lungs, brain, heart, liver, and hind limbs were dissected $24 \mathrm{~h}$ post injection and were subjected to biophotonic imaging assay for evaluation of organ distribution of DiR-Lipo-ZEB1GFP using the IVIS Spectrum In Vivo Imaging System (PekinElmer). The region of interest was circled in the collected tissues and the fluorescence intensity of DiR was measured using Living Image software.

Statistics. Data were presented as mean \pm SD. Statistical analysis was carried out as described in each corresponding figure legend and sample sizes were shown in each corresponding figure legend. $P<0.05$ is considered significant.

Reporting summary. Further information on research design is available in the Nature Research Reporting Summary linked to this article.

\section{Data availability}

The source data underlying Figs. 1b, 1d, 1e, 1g, 1i, 2c, 2d, 2e, 2g, 2h, 2k, 2n, 2p, 2r, 2t, 2v, $3 \mathrm{a}, 3 \mathrm{~d}, 3 \mathrm{e}, 3 \mathrm{~g}, 4 \mathrm{~b}, 4 \mathrm{c}, 4 \mathrm{e}, 4 \mathrm{f}, 4 \mathrm{i}, 5 \mathrm{~d}, 5 \mathrm{f}, 5 \mathrm{~h}, 6 \mathrm{~b}, 6 \mathrm{~d}, 6 \mathrm{f}, 6 \mathrm{~h}, 7 \mathrm{~b}$, and $7 \mathrm{~d}$, and Supplementary Figs. 1c, 1e, 1g, 2b, 2d, 2f, 2h, 3a, 3c, 3f, 3h, 3j, 4b, 4d, 4f, 4h, 5a, 5c, 5d, 6b, 6c, 6d, 6f, and $7 \mathrm{~b}$ are provided as a Source Data file. Unprocessed original scans of blots are shown in Supplementary Fig. 8. The remaining data are contained within the Article, Supplementary Information, or available from the authors upon request. A reporting summary for this Article is available as a Supplementary Information file.

Received: 28 November 2018; Accepted: 11 December 2019; Published online: 23 January 2020

\section{References}

1. Kusumbe, A. P., Ramasamy, S. K. \& Adams, R. H. Coupling of angiogenesis and osteogenesis by a specific vessel subtype in bone. Nature 507, 323-328 (2014).

2. Ramasamy, S. K., Kusumbe, A. P., Wang, L. \& Adams, R. H. Endothelial Notch activity promotes angiogenesis and osteogenesis in bone. Nature 507, 376-380 (2014)

3. Langen, U. et al. Cell-matrix signals specify bone endothelial cells during developmental osteogenesis. Nat. Cell Biol. 19, 189-201 (2017).

4. Xie, H. et al. PDGF-BB secreted by preosteoclasts induces angiogenesis during coupling with osteogenesis. Nat. Med. 20, 1270-1277 (2014).

5. Ellis, S. L. et al. The relationship between bone, hematopoietic stem cells and vasculature. Blood 118, 1516-1524 (2011). 
6. Wang, L. et al. Human type $\mathrm{H}$ vessels are a sensitive biomarker of bone mass. Cell Death Dis. 8, e2760 (2017).

7. Zhao, Q. et al. Mice with increased angiogenesis and osteogenesis due to conditional activation of HIF pathway in osteoblasts are protected from ovariectomy induced bone loss. Bone 50, 763-770 (2012).

8. Xu, R. et al. Targeting skeletal endothelium to ameliorate bone loss. Nat. Med. 24, 823-833 (2018)

9. Heaney, R. P. Pathophysiology of osteoporosis. Endocrinol. Metab. Clin. North Am. 27, 255-265 (1998).

10. Spaderna, S. et al. The transcriptional repressor ZEB1 promotes metastasis and loss of cell polarity in cancer. Cancer Res. 68, 537-544 (2008).

11. Wellner, U. et al. The EMT-activator ZEB1 promotes tumourigenicity by repressing stemness-inhibiting microRNAs. Nat. Cell Biol. 11, 1487-1495 (2009).

12. Zhang, P. et al. ATM-mediated stabilization of ZEB1 promotes DNA damage response and radioresistance through CHK1. Nat. Cell Biol. 16, 864-875 (2014).

13. Vandewalle, C., Van Roy, F. \& Berx, G. The role of the ZEB1 family of transcription factors in development and disease. Cell. Mol. Life Sci. 66, 773-787 (2009).

14. Takagi, T., Moribe, H., Kondoh, H. \& Higashi, Y. DeltaEF1, a zinc finger and homeodomain transcription factor, is required for skeleton patterning in multiple lineages. Development 125, 21-31 (1998).

15. $\mathrm{Fu}, \mathrm{R}$. et al. A ZEB1/p53 signaling axis in stromal fibroblasts promotes mammary epithelial tumours. Nat. Commun. 10, 3210 (2019).

16. Kisanuki, Y. Y. et al. Tie2-Cre transgenic mice: a new model for endothelial cell-lineage analysis in vivo. Dev. Biol. 230, 230-242 (2001).

17. Corada, M. et al. The Wnt/ $\beta$-catenin pathway modulates vascular remodeling and specification by upregulating Dll4/Notch signaling. Dev. Cell 18, 938-949 (2010).

18. Wang, Y. et al. Ephrin-B2 controls VEGF-induced angiogenesis and lymphangiogenesis. Nature 465, 483-486 (2010).

19. Gerber, H. P. et al. VEGF couples hypertrophic cartilage remodeling, ossification and angiogenesis during endochondral bone formation. Nat. Med. 5, 623-628 (1999).

20. Liu, L. et al. ZEB1 is associated with VEGFA expression and blood vessel density in human breast cancer. PLoS ONE 11, e0148774 (2016).

21. Ducy, P., Zhang, R., Geoffroy, V., Ridall, A. L. \& Karsenty, G. Osf2/Cbfa1: a transcriptional activator of osteoblast differentiation. Cell 89, 747-754 (1997).

22. Nakashima, K. et al. The novel zinc finger-containing transcription factor Osterix is required for osteoblast differentiation and bone formation. Cell 108, 17-29 (2002)

23. Millan, J. L. The role of phosphatases in the initiation of skeletal mineralization. Calcif. Tissue Int. 93, 299-306 (2013).

24. Perez-Amodio, S. et al. Endogenous expression and endocytosis of tartrateresistant acid phosphatase (TRAP) by osteoblast-like cells. Bone 36, 1065-1077 (2005).

25. Hsu, D. S. et al. Acetylation of Snail modulates the cytokinome of cancer cells to enhance the recruitment of macrophages. Cancer Cell 26, 534-548 (2014).

26. Hsu, D. S. et al. Regulation of excision repair cross-complementation group 1 by Snail contributes to cisplatin resistance in head and neck cancer. Clin. Cancer Res. 16, 4561-4571 (2010).

27. Hwang, W. L. et al. Snail regulates Interleukin-8 expression, stem cell-like activity, and tumorigenicity of human colorectal carcinoma cells. Gastroenterology 141, 279-291 (2011).

28. Spaderna, S. et al. A transient, EMT-linked loss of basement membranes indicates metastasis and poor survival in colorectal cancer. Gastroenterology 131, 830-840 (2006).

29. Lazarova, D. L., Bordonaro, M. \& Sartorelli, A. C. Transcriptional regulation of the vitamin D3 receptor gene by ZEB. Cell Growth Differ. 12, 319-326 (2001).

30. Rembold, M. et al. A conserved role for Snail as a potentiator of active transcription. Genes Dev. 28, 167-181 (2014).

31. Herranz, N. et al. Polycomb complex 2 is required for E-cadherin repression by the Snaill transcription factor. Mol. Cell. Biol. 28, 4772-4781 (2008).

32. Carlson, M. E., Hsu, M. \& Conboy, I. M. Imbalance between pSmad 3 and Notch induces CDK inhibitors in old muscle stem cells. Nature 454, 528-532 (2008).

33. $\mathrm{Hu}, \mathrm{J}$. et al. Müller glia cells regulate Notch signaling and retinal angiogenesis via the generation of 19,20-dihydroxydocosapentaenoic acid. J. Exp. Med. 211, 281-295 (2014).

34. Potente, M., Gerhardt, H. \& Carmeliet, P. Basic and therapeutic aspects of angiogenesis. Cell 146, 873-887 (2011).

35. Bulter, J. M., Kobayashi, H. \& Rafii, S. Instructive role of the vascular niche in promoting tumour growth and tissue repair by angiocrine factors. Nat. Rev. Cancer 10, 138-146 (2010).

36. Jiang, T. et al. Enhanced transdermal drug delivery by transfersome-embedded oligopeptide hydrogel for topical chemotherapy of melanoma. ACS Nano 12, 9693-9701 (2018).

37. Sun, Q. et al. A collaborative assembly strategy for tumor-targeted siRNA delivery. J. Am. Chem. Soc. 137, 6000-6010 (2015).
38. Assanhou, A. G. et al. Reversal of multidrug resistance by co-delivery of paclitaxel and lonidamine using a TPGS and hyaluronic acid dualfunctionized liposome for cancer treatment. Biomaterials 73, 284-295 (2015).

39. Mora-Raimundo, P., Lozano, D., Manzano, M. \& Vallet-Regí, M. Nanoparticles to knockdown osteoporosis-related gene and promote osteogenic marker expression for osteoporosis treatment. ACS Nano 13, 5451-5464 (2019).

40. Yang, L. et al. Leonurine hydrochloride promotes osteogenic differentiation and increases osteoblastic bone formation in ovariectomized mice by $\mathrm{Wnt} / \beta$ catenin pathway. Biochem. Biophys. Res. Commun. 504, 941-948 (2018).

41. Jing, H. et al. Epigenetic inhibition of Wnt pathway suppresses osteogenic differentiation of BMSCs during osteoporosis. Cell Death Dis. 9, 176 (2018).

42. Phng, L. K. \& Gerhardt, H. Angiogenesis: a team effort coordinated by Notch Dev. Cell 16, 196-208 (2009).

43. Limbourg, F. P. et al. Essential role of endothelial Notch1 in angiogenesis. Circulation 111, 1826-1832 (2005).

44. Benedito, R. et al. Notch-dependent VEGFR3 upregulation allows angiogenesis without VEGF-VEGFR2 signaling. Nature 484, 110-114 (2012).

45. Benedito, R. et al. The notch ligands Dll4 and Jagged1 have opposing effects on angiogenesis. Cell 137, 1124-1135 (2009).

46. Noguera-Troise, I. et al. Blockade of Dll4 inhibits tumor growth by promoting non-productive angiogenesis. Nature 444, 21-28 (2006).

47. Brabletz, S. et al. The ZEB1/miR-200 feedback loop controls Notch signaling in cancer cells. $E M B O$ J. 30, 770-782 (2011).

\section{Acknowledgements}

This research was supported by grants from Natural Science Foundation of China (81973363, 81572745, 91539115, 81603134, 81771985, 81702205, and 81702163), the Jiangsu Provincial Natural Science Funds for Distinguished Young Scholar (BK20170029), the Jiangsu Provincial Natural Science Funds for Young Scholar (BK20160758), the Jiangsu Provincial Innovative Research Program, the State Key Laboratory of Natural Medicines of China Pharmaceutical University (SKLNMZZCX201808), and the "Double First-Class" University project (CPU2018GF02). We thank Dr R. Adams (Max Planck Institute for Molecular Biomedicine, Germany) for providing Cdh5(PAC)-CreERT2 mice and Dr M.-D. Lai (China Pharmaceutical University, China) for critically reviewing this manuscript.

\section{Author contributions}

Z.-Q.W. conceived the project, designed experiments, interpreted results, and wrote the manuscript. R.F. designed and performed experiments, interpreted results, and wrote the manuscript. W.-C.L., Y.X. and M.-Y.G. performed experiments with the help from X.-J.C., N.J., Y.X., L.D. and Q.-Q.Y. T.L., L.-M.W. and R.M. provided relevant advice.

\section{Competing interests}

Z.-Q.W., R.F. and R.M. are inventors on a pending patent related to this work. The other authors declare no competing interests.

\section{Additional information}

Supplementary information is available for this paper at https://doi.org/10.1038/s41467 019-14076-3.

Correspondence and requests for materials should be addressed to Z.-Q.W.

Peer review information Nature Communications thanks Jan Kamps and the other, anonymous, reviewer(s) for their contribution to the peer review of this work. Peer reviewer reports are available.

Reprints and permission information is available at http://www.nature.com/reprints

Publisher's note Springer Nature remains neutral with regard to jurisdictional claims in published maps and institutional affiliations.

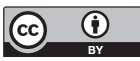

Open Access This article is licensed under a Creative Commons Attribution 4.0 International License, which permits use, sharing, adaptation, distribution and reproduction in any medium or format, as long as you give appropriate credit to the original author(s) and the source, provide a link to the Creative Commons license, and indicate if changes were made. The images or other third party material in this article are included in the article's Creative Commons license, unless indicated otherwise in a credit line to the material. If material is not included in the article's Creative Commons license and your intended use is not permitted by statutory regulation or exceeds the permitted use, you will need to obtain permission directly from the copyright holder. To view a copy of this license, visit http://creativecommons.org/ licenses/by/4.0/.

(c) The Author(s) 2020 